\title{
Design of Optimally Smoothing Multi-Stage Schemes for the Euler Equations
}

\author{
Bram van Leer* \\ Chang-Hsien $\mathrm{Tai}^{\dagger}$ \\ and
}

Kenneth G. Powell ${ }^{\ddagger}$

The University of Michigan

Department of Aerospace Engineering

\begin{abstract}
In this paper, a method is developed for designing multi-stage schemes that give optimal damping of highfrequencies for a given spatial-differencing operator. The objective of the method is to design schemes that combine well with multi-grid acceleration. The schemes are tested on a nonlinear scalar equation, and compared to Runge-Kutta schemes with the maximum stable time-step. The optimally smoothing schemes perform better than the Runge-Kutta schemes, even on a single grid. The analysis is extended to the Euler equations in one space-dimension by use of "characteristic time-stepping," which preconditions the equations, removing stiffness due to variations among characteristic speeds. Convergence rates independent of the number of cells in the finest grid are achieved for transonic flow with and without a shock. Characteristic time-stepping is shown to be preferable to local time-stepping, although use of the optimally damping schemes appears to enhance the performance of local time-stepping. The extension of the analysis to the two-dimensional Euler equations is hampered by the lack of a model for characteristic time-stepping in two dimensions. Some results for local time-stepping are presented.
\end{abstract}

\section{Introduction}

Many aerodynamic problems of current interest hinge on the computation of a steady flow pattern induced by some specific fixed geometry. Numerical methods for such computations can be divided into two classes;

\footnotetext{
*Professor, Member AIAA

† Graduate Research Assistant

'Assistant Professor, Member AIAA
}

explicit and implicit methods. Historically, marching to a steady state has been done primarily by implicit methods, owing to the elliptic nature of the steadyflow equations in the subsonic regime and to influential work on approximate factorization methods done at NASA Ames Research Center in the seventies [1]. Implicit methods are most easily implemented on structured grids, and are notoriously difficult to vectorize and parallelize. They also require substantial storage, but this is less of a restriction given the large amount of memory available with the very newest supercomputers. Explicit strategies require many more iterations than implicit strategies, but each iteration is relatively cheap. Explicit methods also have the advantage of being easily implemented on vector and parallel architectures, and naturally allow local grid refinements [2]. The latter advantage is crucial, since adaptive grid refinement seems to be the most promising way to efficiently obtain spatial accuracy in complex problems.

Explicit methods used in production codes have largely been based on the technique of "local timestepping," according to which each computational cell of fluid is made to evolve with the largest time-step allowed by stability considerations [3]. Mathematically speaking, this corresponds to removing the stiffness due to spatial variations of the flow variables and the grid parameters by a local preconditioning of the residual of the equations by a scalar factor. Since the flow of a compressible fluid is described by a system of equations - $(m+2)$ equations for $m$-dimensional flow - a local matrix preconditioner would be more appropriate. For discrete approximations of the inviscid flow equations, i.e. the Euler equations, this should remove the stiffness due to the differences in the propagation speeds of the various wave modes in the fluid. Analogously to 
local time-stepping, this could be called "characteristic time-stepping." An early example of a preconditioning of this kind was proposed and used by Turkel [4] for subsonic flow computations.

Preconditioning by a local matrix requires, at worst, the similarity transformation of an $(m+2) \times(\dot{m}+2)$ diagonal block in each cell. The computational cost of this may still be considered reasonable within the framework of explicit time-marching, especially if the similarity transformation is further used to improve the accuracy of the spatial discretization.

For one-dimensional inviscid flow, the construction of an effective preconditioner is relatively simple, because the wave speeds and directions are known [5]. In multidimensional flow, the situation is much complicated by the omnidirectional propagation of information: there is no unique decomposition of the flow-field in terms of discrete waves. To find the dominant wave speeds and directions, the local data must be analyzed according to an a priori model; examples of such models are given by Roe [6] and Hirsch et al. [7]. The information extracted can then be incorporated in the discrete residual as well as in the matrix preconditioner. Hirsch's model and characteristic time-stepping have been implemented by Powell and Van Leer [8].

Given the proper local preconditioner, one still has a choice in marching strategy. One approach is to take the maximum allowable time-step with the preconditioned equations, the underlying idea being that the asymptotic steady state will then be reached in fewer steps. Although it is generally agreed that this is the best strategy for single grid computations, it is not necessarily the best strategy for multi-grid computations. In a multi-grid procedure, one special task of the marching scheme is to remove high-frequency components of the error while marching; the multi-grid strategy acts to remove low-frequency components through the use of coarse-grid representations of the solution [9]. In all marching schemes presently in use, the best damping properties are achieved for a time-step that is substantially less than the maximum allowed by stability considerations.

To make a marching scheme a good multi-grid "smoother," the temporal and spatial discretization must be matched to each other. Since the spatial discretization dictates the final accuracy of the solution, the most natural way is to select a spatial discretization and design the time discretization in such a way that short waves are effectively damped. In the multi-grid code developed by Jameson [10], this is achieved by appropriately choosing the values of the parameters of a multi-stage integration method. The analysis on which the choice of parameters is based is strictly scalar and one-dimensional, and is carried out by trial and error. This suggests that a more thorough and comprehensive analysis of the damping properties of multi-stage schemes may lead to improvements in the performance of the multi-grid procedure.

The present research concerns the use of multi-stage methods suitable for multi-grid solution of the Euler equations. The analysis and optimization of the damping properties of such schemes is put on a firm footing (Section 2) and made applicable to systems of equations through use of preconditioning (Section 4); the applicability to multi-dimensional problems is discussed in Section 5. The analysis is illustrated with numerical experiments reported in Sections 3, 4 and 5. In Section 6, results are summarized and future research directions are indicated.

\section{The Multi-Stage Scheme as a High-Frequency Filter}

The generic marching scheme used is a two-stage or predictor-corrector integration method for the linear ordinary differential equation

$$
\frac{d u}{d t}=\lambda u, \quad \lambda \in \mathcal{C},
$$

that is,

$$
\begin{aligned}
\tilde{u} & =u^{n}+\alpha \Delta t \lambda u^{n} \\
u^{n+1} & =u^{n}+\Delta t \lambda \tilde{u} \\
& =\left[1+\lambda \Delta t+\alpha(\lambda \Delta t)^{2}\right] u^{n} .
\end{aligned}
$$

Here the time-step ratio $\alpha$ is a free parameter. As seen from Equation 2c, the stability and damping properties of the scheme are associated with the complex polynomial

$$
P_{2}(z, \alpha)=1+z+\alpha z^{2}, \quad z \equiv \lambda \Delta t .
$$

This polynomial has two complex-conjugate roots, $z_{1}(\alpha)$ and $z_{2}(\alpha)=z_{1}^{*}(\alpha)$, with

$$
z_{1}(\alpha)=-\frac{1}{2 \alpha}-\frac{i}{2 \alpha} \sqrt{4 \alpha-1}
$$

these may be moved along the circle

$$
\left[\Re\left(z_{0}\right)+1\right]^{2}+\left[\Im\left(z_{0}\right)\right]^{2}=1
$$

by varying $\alpha$.

When a partial differential equation is interpreted by the method of lines, $\lambda$ represents the Fourier transform of the spatial differencing operator, and depends on the spatial frequency $\xi$ or, more specifically, on the spatial wave number

$$
\beta=2 \pi \xi \Delta x .
$$

For instance, when solving the convection equation

$$
\frac{\partial u}{\partial t}=-c \frac{\partial u}{\partial x}, \quad c>0,
$$


use of upwind differencing for the spatial derivative gives

$$
\Delta t \frac{\partial u}{\partial t}=-\nu[u(x, t)-u(x-\Delta x, t)],
$$

where the non-dimensional time step,

$$
\nu=\frac{c \Delta t}{\Delta x}
$$

is the Courant-Friedrichs-Lewy (CFL) number. After inserting harmonic data

$$
u(x)=u_{0} e^{-2 \pi i \xi x},
$$

Equation 6 reduces to Equation 1 with

$$
\lambda \Delta t \equiv z(\beta, \nu)=-\nu\left(1-e^{-i \beta}\right) .
$$

The key observation to be made here is that, for any $\beta_{0}$ in the high-frequency range $[\pi / 2, \pi]$, it is possible to make $z\left(\beta_{0}, \nu\right) \equiv \lambda\left(\beta_{0}\right) \Delta t$ coincide with a zero of $P_{2}(z, \alpha)$, by choosing a particular combination of $\alpha$ and $\Delta t$. This results in perfect damping of the wave with wave number $\beta_{0}$ in one application of the predictorcorrector scheme. Using strings of predictor-corrector schemes, tuned to damp different frequencies, the entire high-frequency range can be damped to arbitrarily low levels.

Strings of predictor-corrector methods generate multi-stage methods with an even number of stages; to get an odd number of stages, a single application of the "forward-Euler" scheme

$$
\begin{aligned}
u^{n+1} & =u^{n}+\Delta t \lambda u^{n} \\
& =(1+\lambda \Delta t) u^{n},
\end{aligned}
$$

should be included in the string. The forward-Euler step has amplification factor

$$
P_{1}(z)=1+z
$$

this polynomial has one zero, at

$$
z_{1}=-1 \text {. }
$$

A second key observation is that, for any fixed number of stages, there is an optimum scheme, in the $L_{\infty}$ sense, reducing all of the high frequencies to an amplitude not exceeding a unique minimal threshold level. It is these "optimally smoothing" multi-stage schemes that are developed here, for use in multi-grid Euler codes.

\subsection{An Example: Optimal Two-Stage Scheme for Upwind Differencing}

In this section, the construction of the optimally smoothing two-stage scheme for the integration of
Equation 6 is derived, with $z(\beta, \nu)$ given by Equation 9 . Note that $z(\beta, \nu)$ traces the circle

$$
(\Re(z)+\nu)^{2}+(\Im(z))^{2}=\nu^{2}
$$

as $\beta$ is varied; for

$$
\nu=\nu_{0}=1
$$

this circle is also the locus of the zeroes of $P_{2}(z, \alpha)$. Thus, for a frequency to be perfectly damped, we must set $z_{1}(\alpha)=z\left(\beta_{0}, \nu_{0}\right)$, or

$$
-\frac{1}{2 \alpha}-\frac{i}{2 \alpha} \sqrt{4 \alpha-1}=-\left(1-e^{-i \beta_{0}}\right),
$$

from which follows the proper choice of $\alpha$ :

$$
\alpha=\alpha_{0}=\frac{1}{2\left(1-\cos \beta_{0}\right)} .
$$

Note that the "aliased" frequency, with wave number $-\beta_{0}$, corresponds to $z_{1}^{*}$ and is therefore also perfectly damped.

The optimal value of $\beta_{0} \in[\pi / 2, \pi]$, namely, the value that minimizes the maximum of $\left|P_{2}\left(z\left(\beta, \nu_{0}\right), \alpha\right)\right|$ for $\beta \in[\pi / 2, \pi]$, may now be found. On the circle defined in Equation $3 \mathrm{c}$, the polynomial $P_{2}\left(z\left(\beta, \nu_{0}\right), \alpha_{0}\right)$ takes the value

$$
\begin{aligned}
P_{2}\left(z\left(\beta, \nu_{0}\right), \alpha_{0}\right) & \equiv P_{2}(\beta) \\
& =\frac{\cos \beta-\cos \beta_{0}}{1-\cos \beta_{0}} e^{-i \beta},
\end{aligned}
$$

leading to

$$
\left|P_{2}(\beta)\right|=\frac{\left|\cos \beta-\cos \beta_{0}\right|}{1-\cos \beta_{0}} .
$$

This function has local extrema only at the intervalends $\beta=\pi / 2$ and $\beta=\pi$; the equal-excursion principle dictates that the smallest range of values is obtained when the endpoint values are equal, i.e.

$$
\left|P\left(\frac{\pi}{2}\right)\right|=|P(\pi)|
$$

or

$$
\frac{1+\cos \beta_{0}}{1-\cos \beta_{0}}=\frac{-\cos \beta_{0}}{1-\cos \beta_{0}}, \quad \beta_{0} \in\left[\frac{\pi}{2}, \pi\right],
$$

yielding

$$
\cos \beta_{0}=-\frac{1}{2}
$$

or

$$
\beta_{0}=\frac{2 \pi}{3},
$$

and

$$
\begin{aligned}
\alpha_{0} & =\frac{1}{3}, \\
\max _{\beta \in[\pi / 2, \pi]}\left|P_{2}(\beta)\right| & =\frac{1}{3} .
\end{aligned}
$$




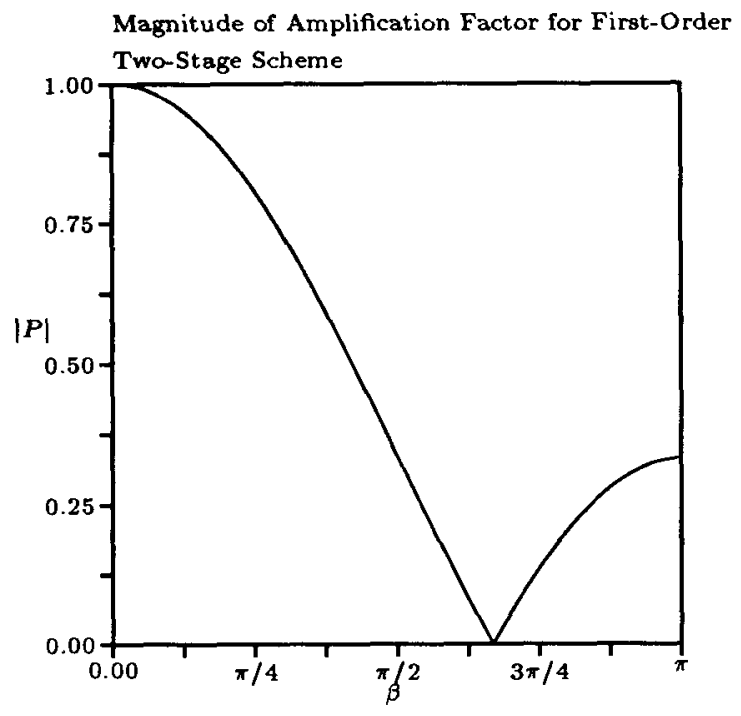

Figure 1: Magnitude of amplification factor as a function of wave number, for multi-stage scheme with optimal high-frequency smoothing. First-order spatial differencing, two-stage scheme.

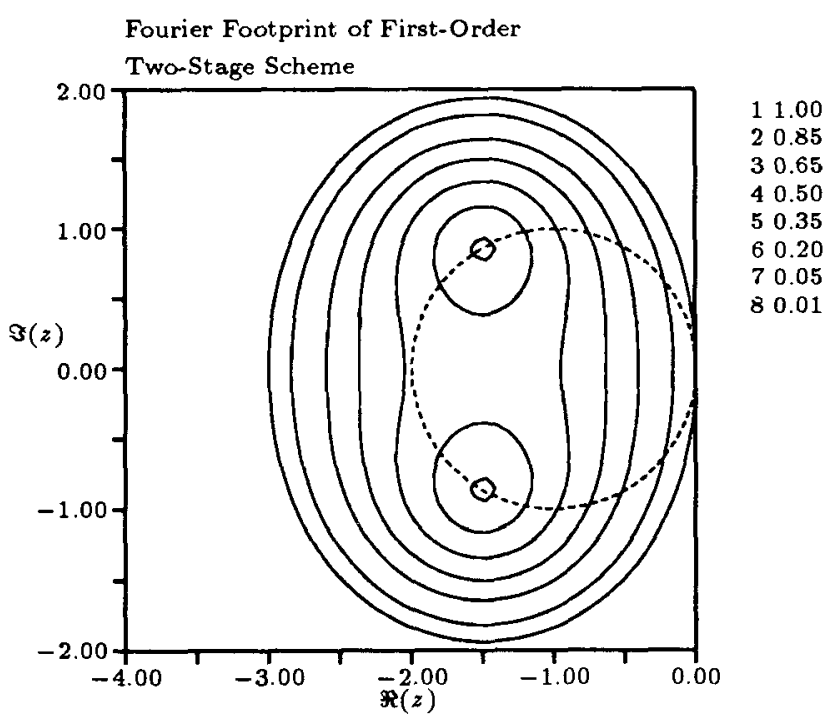

Figure 2: Locus of Fourier transform of spatialdifferencing operator (dashed line), and contours of magnitude of amplification factor of marching scheme. First-order, two-stage scheme
Magnitude of Amplification Factor for First-Order One-Stage Scheme

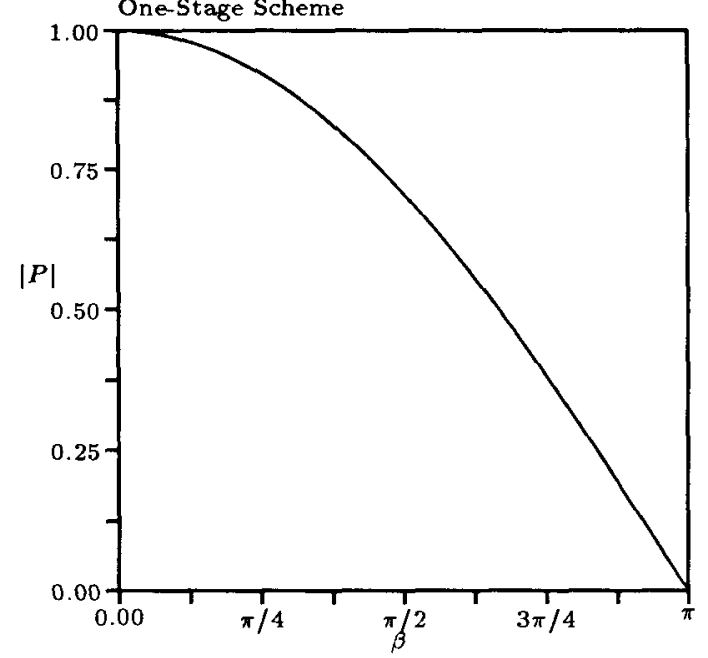

Figure 3: Amplification factor; first-order, one-stage scheme

A graphic representation of $\left|P_{2}(\beta)\right|$ is given in Figure 1; the contours of $\left|P_{2}\left(z, \alpha_{0}\right)\right|$ together with the locus of $z\left(\beta, \nu_{0}\right)$ are shown in Figure 2 .

The analysis for the single-stage scheme, Equation $10 \mathrm{~b}$, is trivial, but given below for completeness. The associated polynomial is given in Equation 11. For a frequency to be perfectly damped, $z_{1}$ is again set equal to $z(\beta, \nu)$, as given in Equation 9, i.e.,

$$
-1=-\nu\left(1-e^{-i \beta}\right)
$$

This yields

$$
\begin{aligned}
& \beta=\beta_{0}=\pi, \\
& \nu=\nu_{0}=\frac{1}{2} .
\end{aligned}
$$

On the circle with radius $\nu_{0}=1 / 2$, traced by $z\left(\beta, \nu_{0}\right)$ (cf. Equation 13), $P_{1}(z)$ takes the value

$$
\begin{aligned}
P_{1}\left(z\left(\beta, \nu_{0}\right)\right) \equiv P_{1}(\beta) & =\frac{1}{2}+\frac{1}{2} e^{-i \beta} \\
& =\cos \left(\frac{\beta}{2}\right) e^{-i \frac{\beta}{2}},
\end{aligned}
$$

yielding

$$
\max _{\beta \in[\pi / 2, \pi]}\left|P_{1}(\beta)\right|=\cos \frac{\pi}{4}=\frac{\sqrt{2}}{2}
$$

A graphic representation of $\left\{P_{1}(\beta)\right\}$ is given in Figure 3 ; the contours of $\left|P_{1}(z)\right|$ together with the locus of $z\left(\beta, \nu_{0}\right)$ are shown in Figure 4. 


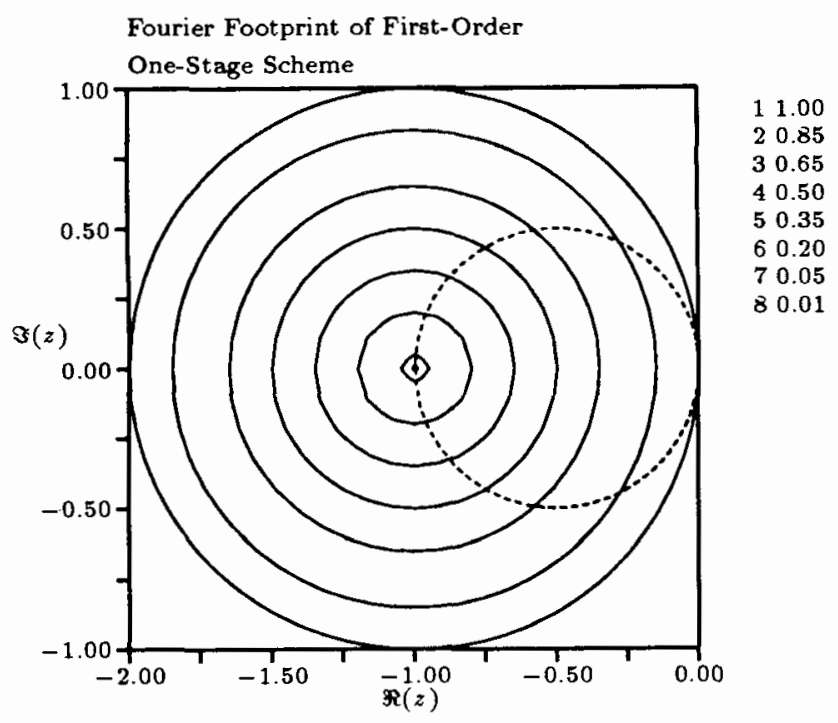

Figure 4: Locus and contours; first-order, one-stage scheme

\subsection{Optimal Multi-Stage Schemes for Upwind Differencing}

When applying a sequence of $m$ predictor-corrector methods, each annihilating a high frequency $\beta_{k}$ through the use of

$$
\begin{aligned}
\nu_{k} & =1 \\
\alpha_{k} & =\frac{1}{2\left(1-\cos \beta_{k}\right)},
\end{aligned}
$$

the product scheme corresponds to the polynomial

$$
P_{2 m}\left(z, \alpha_{1}, \ldots, \alpha_{m}\right)=\prod_{k=1}^{m}\left(1+z+\alpha_{k} z^{2}\right)
$$

where $z$ corresponds to a CFL number of 1 ; inserting $z\left(\beta, \nu_{k}\right)$ yields

$$
\begin{aligned}
\left|P_{2 m}\left(z, \alpha_{1}, \ldots, \alpha_{m}\right)\right| & \equiv\left|P_{2 m}(\beta)\right| \\
& =\left|\prod_{k=1}^{m} \frac{\cos \beta-\cos \beta_{k}}{1-\cos \beta_{k}}\right|
\end{aligned}
$$

The product on the right-hand side of Equation $30 \mathrm{~b}$ is an $m^{\text {th }}$ degree polynomial in $\cos \beta$. To achieve the smallest range of values of $P_{2 m}(\beta)$ as well as $\left|P_{2 m}(\beta)\right|$ on the inverval $[\pi / 2, \pi]$, the polynomial must be one that reduces to the Chebyshev polynomial $T_{m}(y)$, after a mapping of its useful domain, and a scale transformation. In particular, by mapping $\cos \beta$, for $\beta \in[\pi / 2, \pi]$, onto $y$, for $y \in[-1,1]$, according to

$$
\cos \beta=\frac{1}{2}(y-1)
$$

one obtains

$$
\begin{aligned}
\left|P_{2 m}(\beta(y))\right|= & \left|\prod_{k=1}^{m} \frac{y-y_{k}}{3-y_{k}}\right| \\
= & \left|C\left(y_{1}, \ldots, y_{m}\right) T_{m}(y)\right|, \\
& -1 \leq y \leq 1
\end{aligned}
$$

where $C\left(y_{1}, \ldots, y_{m}\right)$ is a scale factor. It follows that the $y_{k}$ must be the zeroes of $T_{m}(y)$, that is,

$$
y_{k}=\cos \left(\frac{(2 k-1) \pi}{2 m}\right), \quad k=1, \ldots, m
$$

this leads to

$$
\beta_{k}=\arccos \left(\frac{y_{k}-1}{2}\right) \text {. }
$$

The maximum of $\left|P_{2 m}(\beta)\right|$ on $[\pi / 2, \pi]$ may now be calculated by inserting $y=1$ into Equation $32 \mathrm{a}$, i.e.

$$
\left|P_{2 m}\right|_{\max } \equiv \max _{\beta \in[\pi / 2, \pi]}\left|P_{2 m}(\beta)\right|=\prod_{k=1}^{m} \frac{1-y_{k}}{3-y_{k}}
$$

For example, the amplification factor of the optimal four-stage scheme $(m=2)$ has zeroes at

$$
\begin{aligned}
& y_{1}=\cos \frac{\pi}{4}=\frac{\sqrt{2}}{2} \\
& y_{2}=\cos \frac{3 \pi}{4}=-\frac{\sqrt{2}}{2},
\end{aligned}
$$

from which follows

$$
\begin{gathered}
\beta_{1}=\arccos \left(-\frac{1}{2}+\frac{\sqrt{2}}{4}\right)=0.54678 \pi \\
\beta_{2}=\arccos \left(-\frac{1}{2}-\frac{\sqrt{2}}{4}\right)=0.82556 \pi \\
\alpha_{1}=\frac{2}{6-\sqrt{2}}, \\
\alpha_{2}=\frac{2}{6+\sqrt{2}},
\end{gathered}
$$

and

$$
\left|P_{4}(\beta)\right|_{\max }=\frac{1}{17}
$$

The damping properties of this scheme are illustrated in Figures 5 and 6 . The amplification factor of a fourstage, first-order scheme in which the zeroes are nonoptimally placed is shown in Figure 7 , for comparison. Note that the value of $|P|$ at $\beta=\pi / 2$ in the non-optimal case is greater than the minimax value of Equation 39 .

For an odd number of stages, e.g. $2 m+1$, achieved by a sequence of $m$ predictor-corrector schemes with a 


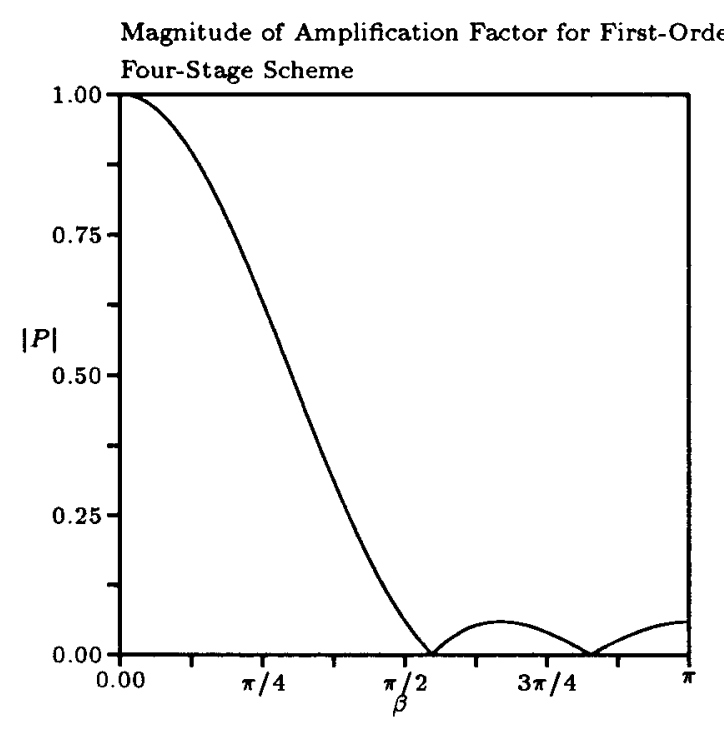

Figure 5: Amplification factor; first-order, four-stage scheme

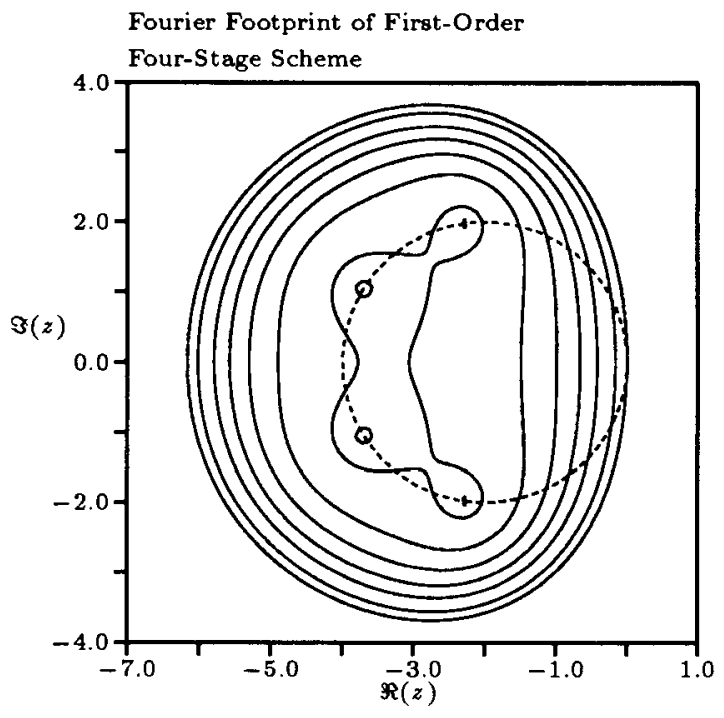

Figure 6: Locus and contours; first-order, four-stage scheme

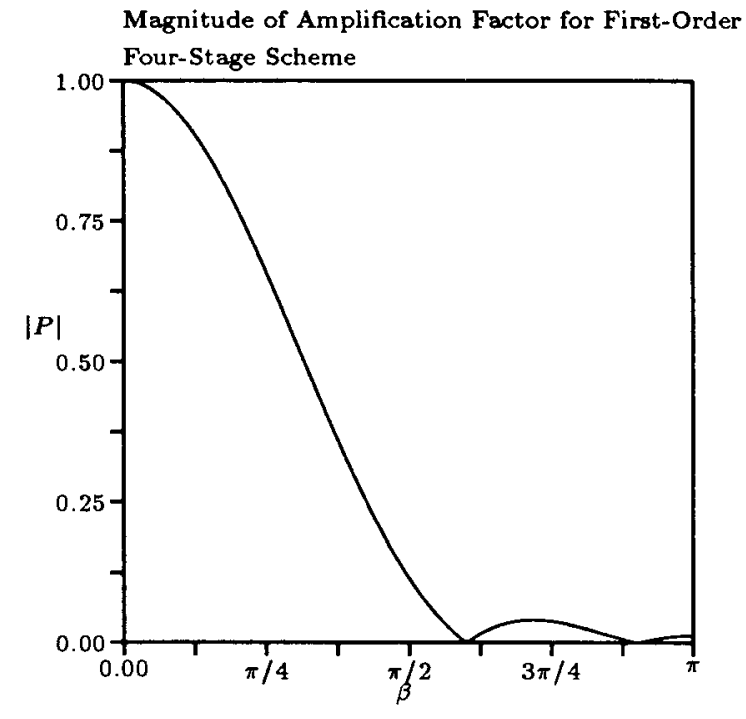

Figure 7: Amplification factor for non-optimal firstorder, four-stage scheme

CFL number of 1 and a single forward-Euler step with CFL number $1 / 2$, the associated polynomial is

$$
P_{2 m+1}(z)=\left(1+\frac{z}{2}\right) \prod_{k=1}^{m}\left(1+z+\alpha_{k} z^{2}\right),
$$

where $z$ corresponds to a CFL number of 1 ; combining Equations 18 and $26 \mathrm{~b}$ yields

$$
\begin{aligned}
\left|P_{2 m+1}(\beta)\right| & =\left|\cos \frac{\beta}{2} \prod_{k=1}^{m} \frac{\cos \beta-\cos \beta_{k}}{1-\cos \beta_{k}}\right| \\
& =\left|\cos \frac{\beta}{2} \prod_{k=1}^{m} \frac{\cos ^{2} \frac{\beta}{2}-\cos ^{2} \frac{\beta_{k}}{2}}{\left(1-\cos ^{2} \frac{\beta_{k}}{2}\right)}\right| .
\end{aligned}
$$

The expression between modulus bars is now a polynomial of order $2 m+1$ in $\cos (\beta / 2)$; for $\beta \in[\pi / 2, \pi]$ its argument runs from $\sqrt{2} / 2$ to 0 . This polynomial will have the smallest range for $\beta \in[\pi / 2, \pi]$ if it can be reduced to the Chebyshev polynomial $T_{2 m+1}(y)$. To achieve this, the mapping

$$
\cos \frac{\beta}{2}=\frac{\sqrt{2}}{2} y
$$

is used, which leads to

$$
\begin{aligned}
\left|P_{2 m+1}(\beta(y))\right|= & \frac{\sqrt{2}}{2}\left|y \prod_{k=1}^{m} \frac{y^{2}-y_{k}^{2}}{2-y_{k}^{2}}\right| \\
= & \left|C\left(y_{1}, \ldots, y_{m}\right) T_{2 m+1}(y)\right|, \\
& 0 \leq y \leq 1 .
\end{aligned}
$$

Note that only half of the domain of $T_{2 m+1}(y)$ is used. 


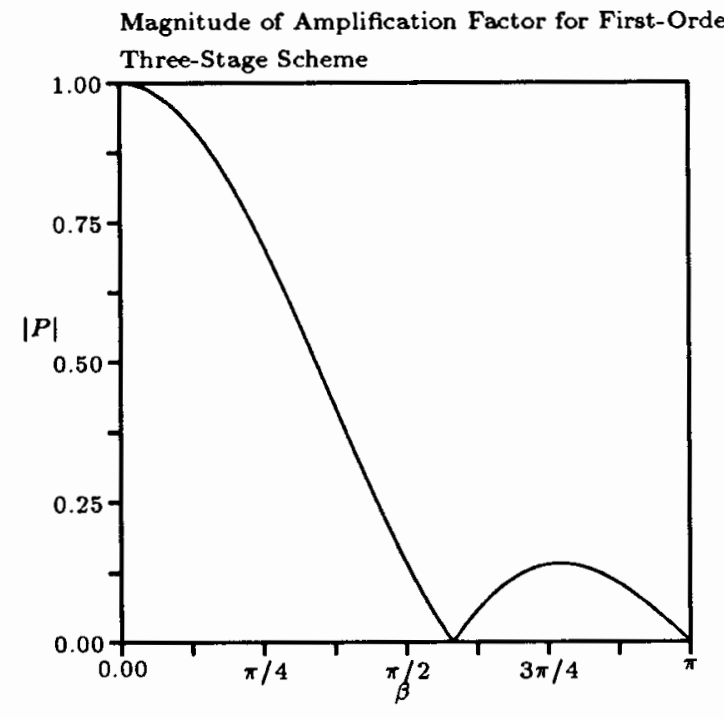

Figure 8: Amplification factor; first-order, three-stage scheme

In this case, the $y_{k}$ span the first $m$ zeroes of $T_{2 m+1}(y)$ :

$$
y_{k}=\cos \left(\frac{(2 k-1) \pi}{2(2 m+1)}\right), \quad k=1, \ldots, m
$$

As before, inserting $y=1$ into Equation 43a yields the minimized maximum amplification factor for the high frequencies:

$$
\left|P_{2 m+1}(\beta)\right|_{\max }=\frac{\sqrt{2}}{2} \prod_{k=1}^{m} \frac{1-y_{k}^{2}}{2-y_{k}^{2}}
$$

For example, the amplification factor of the threestage scheme $(m=1)$ has a zero at

$$
y=\cos \frac{\pi}{6}=\frac{\sqrt{3}}{2},
$$

apart from the zero at $y=0$ (i.e., $\beta=\pi$ ). This gives

$$
\begin{gathered}
\beta_{1}=2 \arccos \frac{\sqrt{6}}{4}=0.58043 \pi, \\
\alpha_{1}=\frac{2}{5}
\end{gathered}
$$

and

$$
\left|P_{3}(\beta)\right|_{\max }=\frac{\sqrt{2}}{10} .
$$

The damping properties of this scheme are illustrated in Figures 8 and 9.

Five- and six-stage schemes can be built analogously. The damping properties of the five-stage scheme are shown in Figures 10 and 11; the properties of the sixstage scheme are shown in Figures 12 and 13.

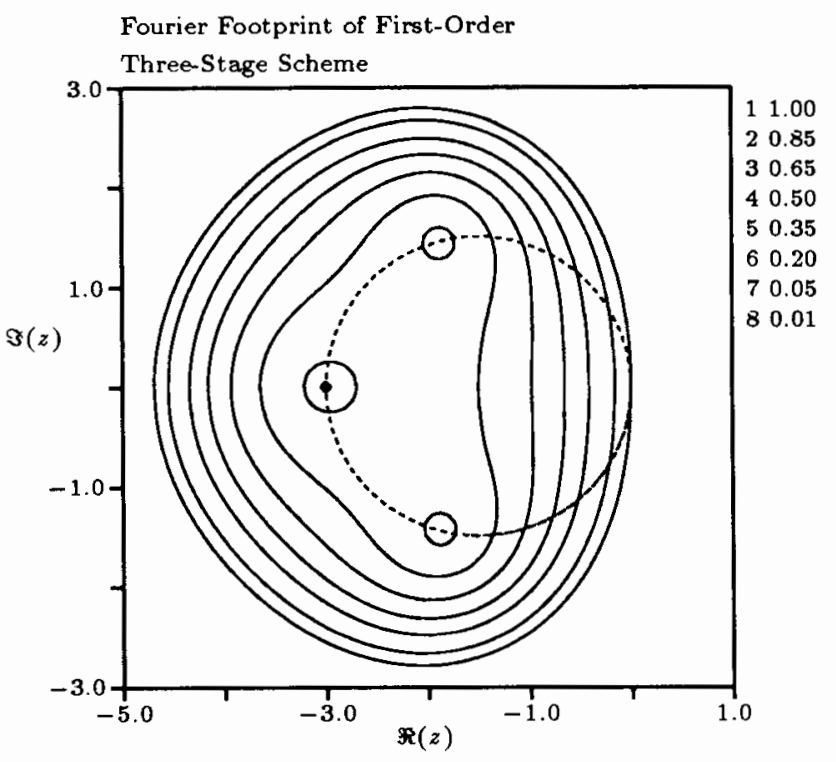

Figure 9: Locus and contours; first-order, three-stage scheme

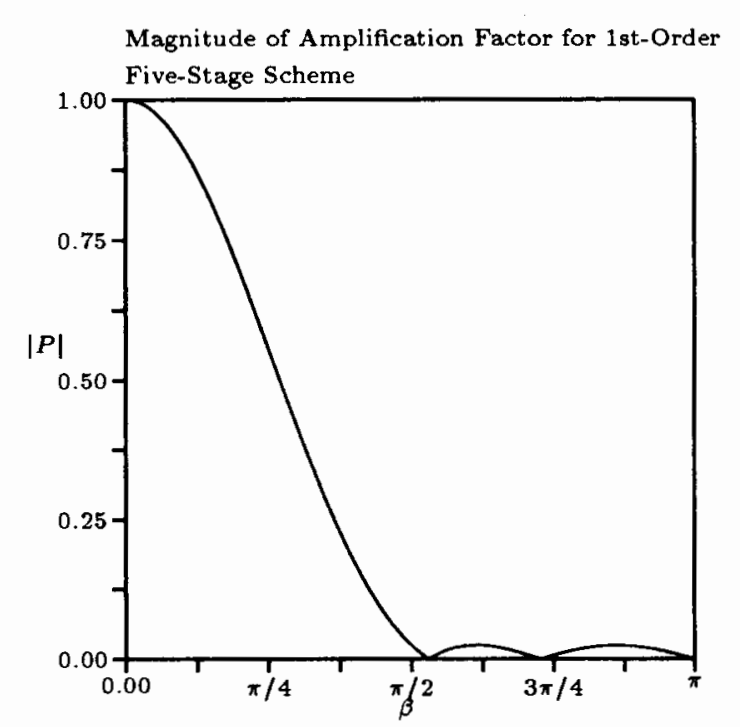

Figure 10: Amplification factor; first-order, five-stage scheme 


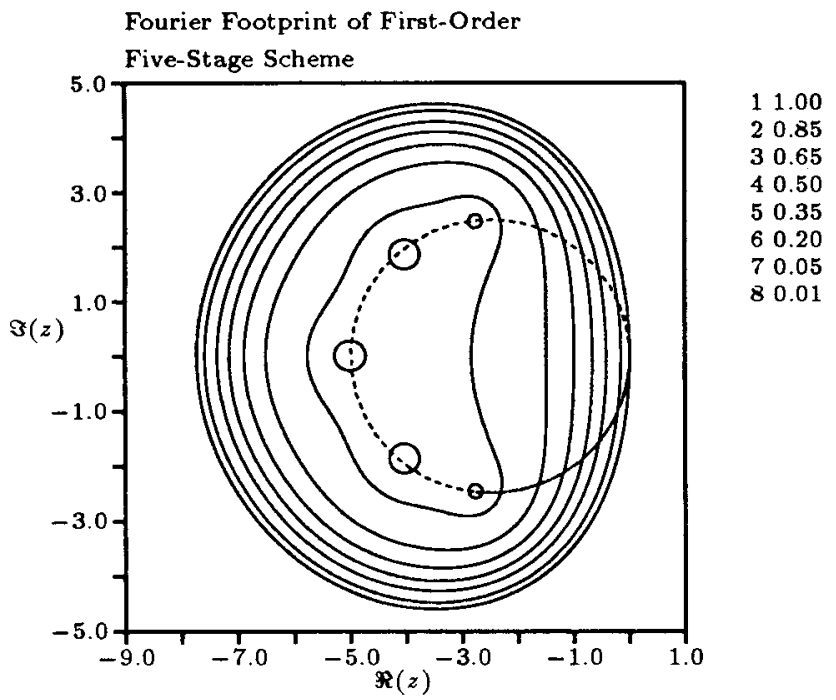

Figure 11: Locus and contours; first-order, five-stage scheme

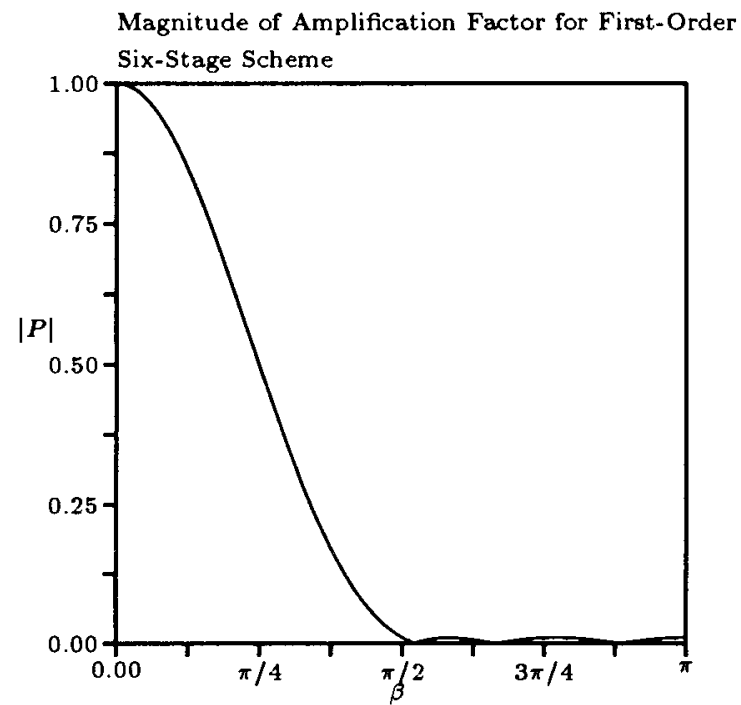

Figure 12: Amplification factor; first-order, six-stage scheme

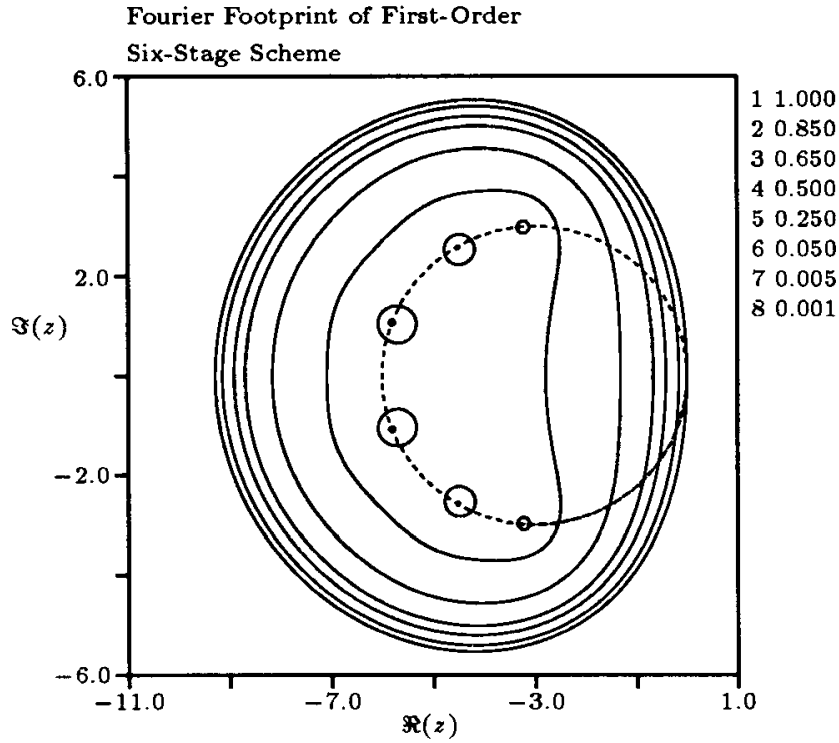

Figure 13: Locus and contours; first-order, six-stage scheme

\subsection{Optimal Multi-Stage Schemes for General Spatial Differencing}

For a general spatial-differencing operator, whether convective or diffusive, or a combination of both, the Fourier transform can still be written as

$$
\lambda \Delta t \equiv z(\beta, \nu)=\nu[a(\beta)+i b(\beta)],
$$

where $\nu$ is a nondimensional time-step; due to a preoccupation with convective equations $\nu$ shall continue to be referred to as the CFL number. Repeating the two-stage analysis of Section 2.1 yields

$$
\begin{aligned}
& \alpha_{0}=\frac{a_{0}^{2}+b_{0}^{2}}{4 a_{0}^{2}}, \\
& \nu_{0}=\frac{2\left|a_{0}\right|}{a_{0}^{2}+b_{0}^{2}},
\end{aligned}
$$

where $a_{0} \equiv a\left(\beta_{0}\right), b_{0} \equiv b\left(\beta_{0}\right)$, for the predictorcorrector scheme that annihilates the high frequency $\beta_{0}$. The single-step scheme can only damp the frequency for which the Fourier transform (Equation 50) is realvalued; for any finite-difference operator this means

$$
\beta_{0}=\pi .
$$

The corresponding CFL number is

$$
\nu_{0}=\frac{1}{\left|a_{0}\right|} \text {. }
$$

For the more complex differencing operators, analytic optimization of the high-frequency damping in a string of predictor-corrector and single-step operators is no 
longer feasible. An iterative method for solving this minimax problem is described below.

Suppose that, in optimizing an $m$-stage method ( $m$ even or odd), as an initial guess or from the previous iteration, a set of perfectly damped frequencies $\beta_{k}$, $k=1, \ldots, \hat{m}$, with $\hat{m}=\operatorname{int}(m / 2)$, has been obtained; let these be represented by the vector $\boldsymbol{\beta}_{0}$. There are $\hat{m}+1$ local maxima of $\left|P_{m}(\beta)\right|$ on the interval $[\pi / 2, \pi]$, called $M_{k}, k=1, \ldots, \hat{m}+1$; these can be found by a numerical search. As long as these values are not equal, the scheme is not optimal. The lack of equality is expressed in the form of an $L_{2}$-residual

$$
R_{0} \equiv R\left(\boldsymbol{\beta}_{0}\right)=\sum_{k=1}^{\hat{m}}\left(M_{k+1}\left(\boldsymbol{\beta}_{0}\right)-M_{k}\left(\boldsymbol{\beta}_{0}\right)\right)^{2},
$$

which is then brought closer to zero by one step of a Newton process. Since $\hat{m}$ frequencies must be updated, $\hat{m}$ independent residual values must be obtained. For this purpose, all frequencies $\beta_{k}$ are perturbed by a small amount $\delta \beta$, i.e.,

$$
\beta_{k}^{\prime}=\beta_{k}+\delta \beta,
$$

and $\hat{m}$ new frequency vectors $\boldsymbol{\beta}^{(i)}$ are formed such that the first $i$ frequencies are perturbed, i.e.:

$$
\begin{aligned}
\boldsymbol{\beta}^{(i)}= & \left(\beta_{1}^{\prime}, \ldots, \beta_{i}^{\prime}, \beta_{i+1}, \ldots, \beta_{\hat{m}}\right), \\
& i=1, \ldots, \hat{m} .
\end{aligned}
$$

For all of these frequency vectors, residuals are obtained in the manner described above; these are called $R^{(i)}$,

$$
R^{(i)} \equiv R\left(\boldsymbol{\beta}^{(i)}\right), \quad i=1, \ldots, \hat{m},
$$

and are collectively indicated by the residual vector $\boldsymbol{R}$ :

$$
\boldsymbol{R} \equiv\left(R^{(1)}, \ldots, R^{(\hat{m})}\right) .
$$

The Jacobian matrix $\mathbf{J} \equiv d \boldsymbol{R}(\boldsymbol{\beta}) / d \boldsymbol{\beta}$ of the residual vector with respect to the frequency vector, needed for a Newton step, is computed approximately by means of finite-differencing. Define the vectors $\beta^{(i, j)}$ as $\beta^{(i)}$ with the $j^{\text {th }}$ component either perturbed or 'unperturbed', i.e.

$$
\begin{aligned}
\boldsymbol{\beta}^{(i, j)}= & \left(\beta_{1}^{\prime}, \ldots, \beta_{j-1}^{\prime}, \beta_{j}, \beta_{j+1}^{\prime}, \ldots, \beta_{i}^{\prime}, \beta_{i+1}, \ldots, \beta_{\dot{m}}\right), \\
& j \leq i, \\
\boldsymbol{\beta}^{(i, j)}= & \left(\beta_{1}^{\prime}, \ldots, \beta_{i}^{\prime}, \beta_{i+1}, \ldots, \beta_{j-1}, \beta_{j}^{\prime}, \beta_{j+1}, \ldots, \beta_{\dot{m}}\right), \\
& j>i,
\end{aligned}
$$

and define the corresdponding residuals $R^{(i, j)}$,

$$
R^{(i, j)} \equiv R\left(\boldsymbol{\beta}^{(i, j)}\right)
$$

some of which have been calculated previously; the elements of the Jacobian are then evaluated according to

$$
\begin{aligned}
& J_{k \ell}= \frac{\partial R^{(k)}}{\partial \beta_{\ell}} \\
&= \frac{R^{(k)}-R^{(k, \ell)}}{\delta \beta}, \\
& \ell \leq k ; \\
& J_{k \ell=} \frac{R^{(k, \ell)}-R^{(k)}}{\delta \beta}, \\
& \ell>k .
\end{aligned}
$$

The Newton step amounts to solving for the correction vector $\Delta \boldsymbol{\beta}$ from

$$
-\mathbf{J} \Delta \boldsymbol{\beta}=\mathbf{R},
$$

and updating $\boldsymbol{\beta}_{0}$ :

$$
\boldsymbol{\beta}_{0}:=\boldsymbol{\beta}_{0}+\Delta \boldsymbol{\beta} .
$$

The above procedure was implemented to find the first six multi-stage schemes with optimal highfrequency damping for the spatial differencing operator with Fourier transform

$$
\begin{aligned}
\lambda \Delta t=-\nu & \left(1-\exp ^{-i \beta}\right)\{1 \\
& +\frac{1-\kappa}{4}\left(1-\exp ^{-i \beta}\right) \\
& \left.+\frac{1+\kappa}{4}\left(\exp ^{i \beta}-1\right)\right\},
\end{aligned}
$$

corresponding to higher-order upwind-biased differencing [11]. The parameter $\kappa$ regulates the upwind bias: $\kappa=1$ yields central differencing, $\kappa=-1$ second-orderaccurate fully upwind differencing, $\kappa=1 / 3$ third-orderaccurate upwind-biased differencing. For this latter choice, the damping properties of the six schemes are displayed in Figures 14 to 24.

The important parameters of the first six multi-stage schemes for several spatial-differencing operators are listed in Tables 1-5; these require some explanation. Specifically, the quantities $\alpha_{k}$ listed in Tables 1-3 are not the time-step ratios of the constituent predictorcorrector schemes, but the time-step ratios arising in the practical implementation of an $m$-stage scheme, i.e.,

$$
\begin{aligned}
u^{(0)} & =u^{n} \\
u^{(k)} & =u^{(0)}+\alpha_{k} \Delta t \lambda u^{(k-1)}, k=1, \ldots, m, \\
u^{n+1} & =u^{(m)}
\end{aligned}
$$

Note that the $m^{\text {th }}$ step always spans the full timeinterval $\Delta t$, so that

$$
\alpha_{m}=1
$$




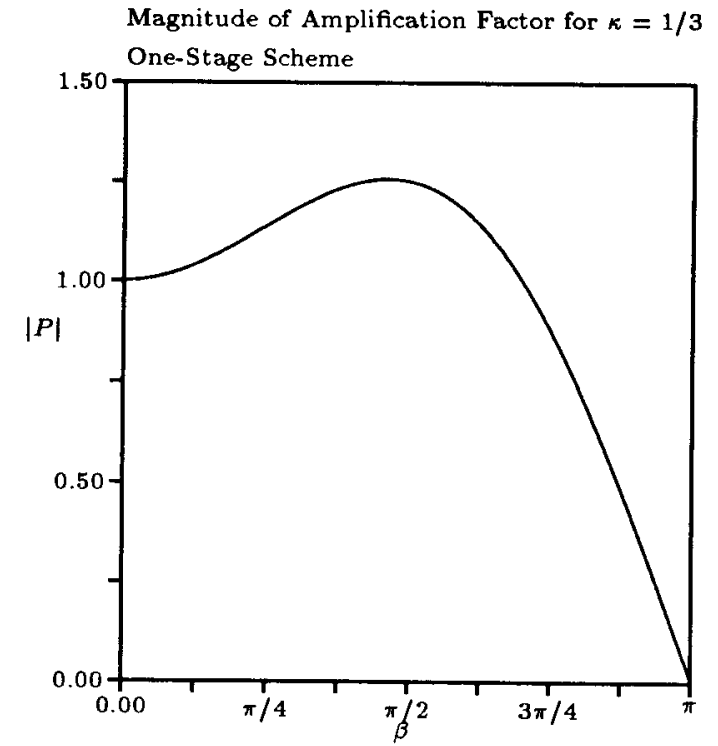

Figure 14: Amplification factor; third-order, one-stage scheme

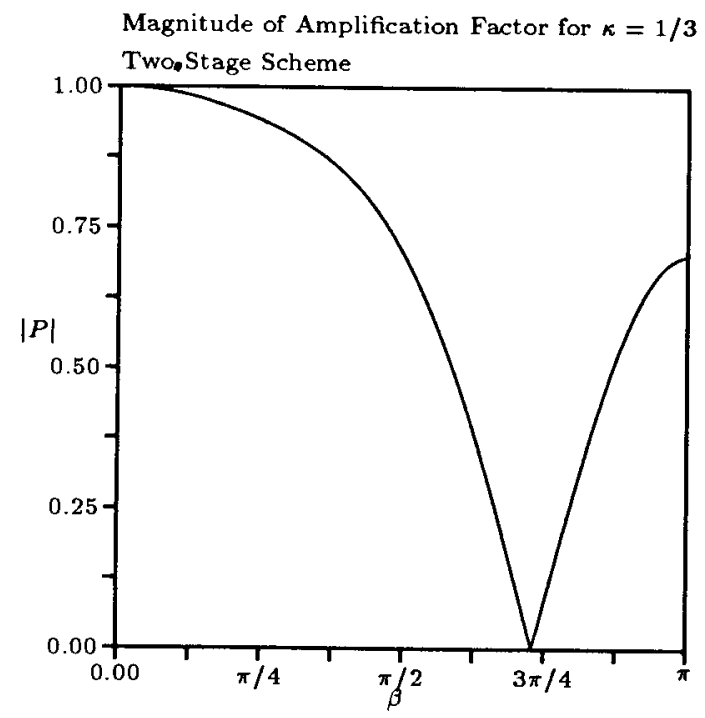

Figure 15: Amplification factor; third-order, two-stage scheme

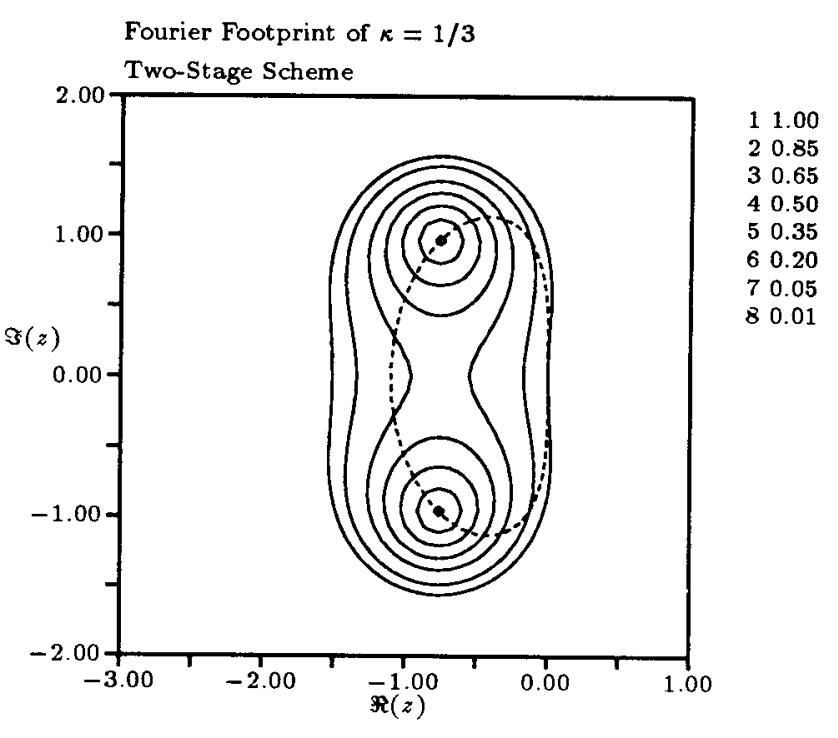

Figure 16: Locus and contours; third-order, two-stage scheme

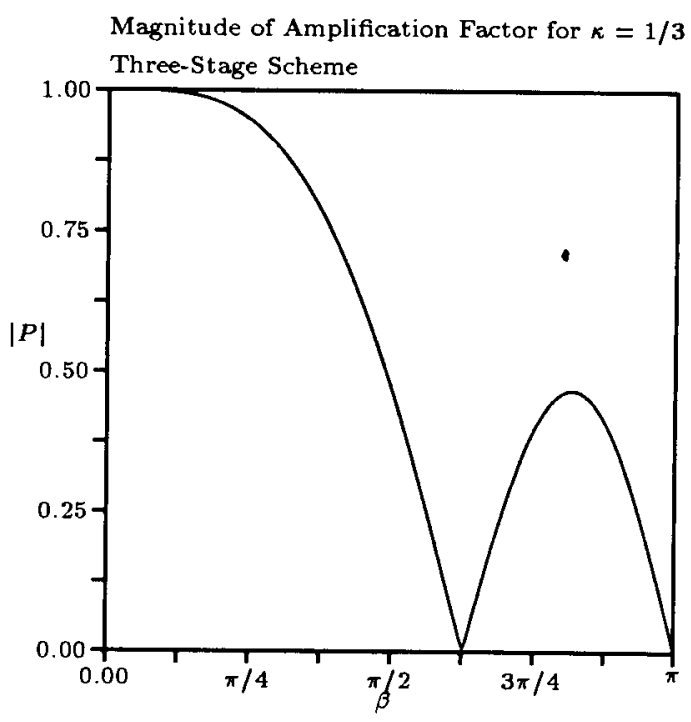

Figure 17: Amplification factor; third-order, threestage scheme 


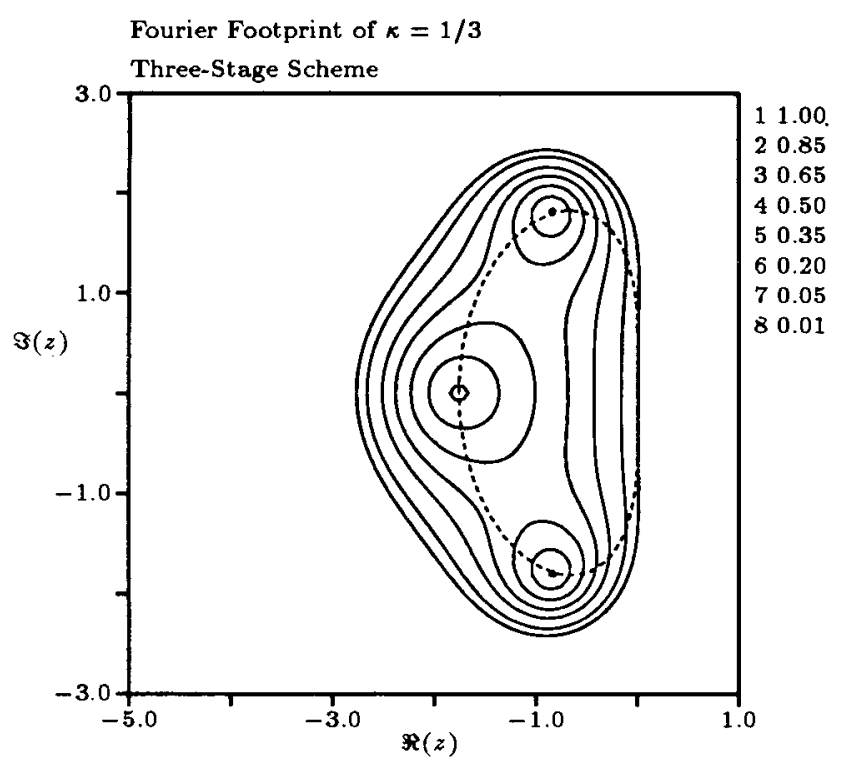

Figure 18: Locus and contours; third-order, three-stage scheme

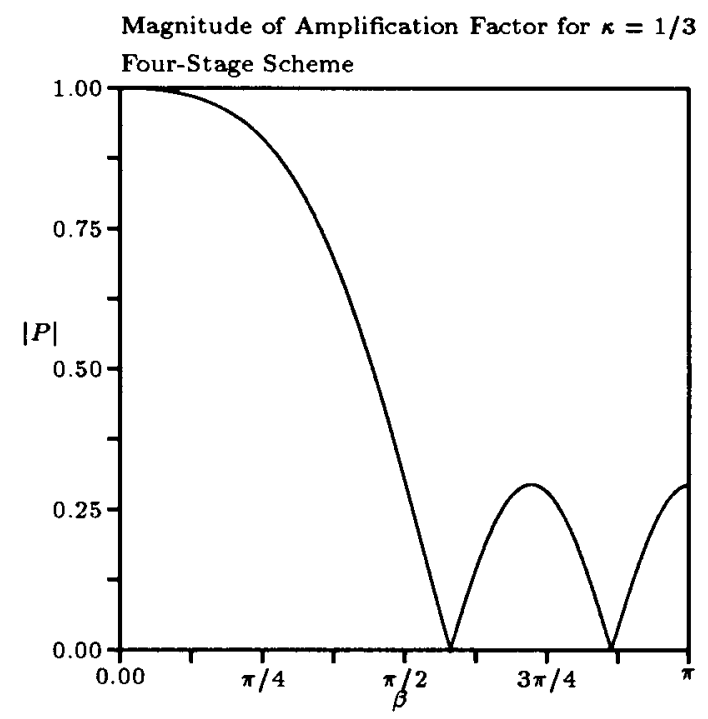

Figure 19: Amplification factor; third-order, four-stage scheme

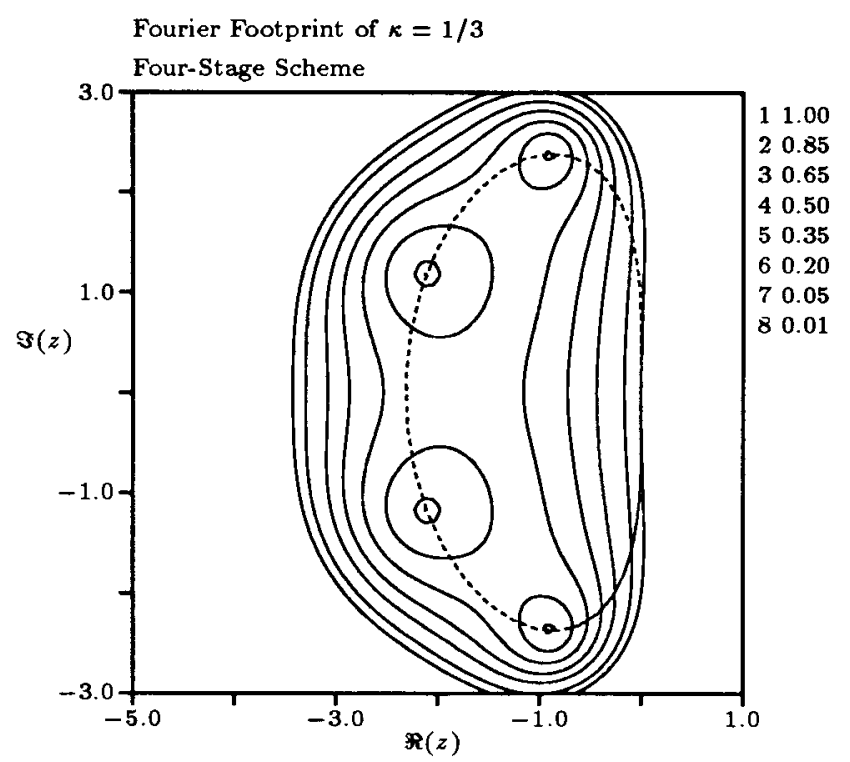

Figure 20: Locus and contours; third-order, four-stage scheme

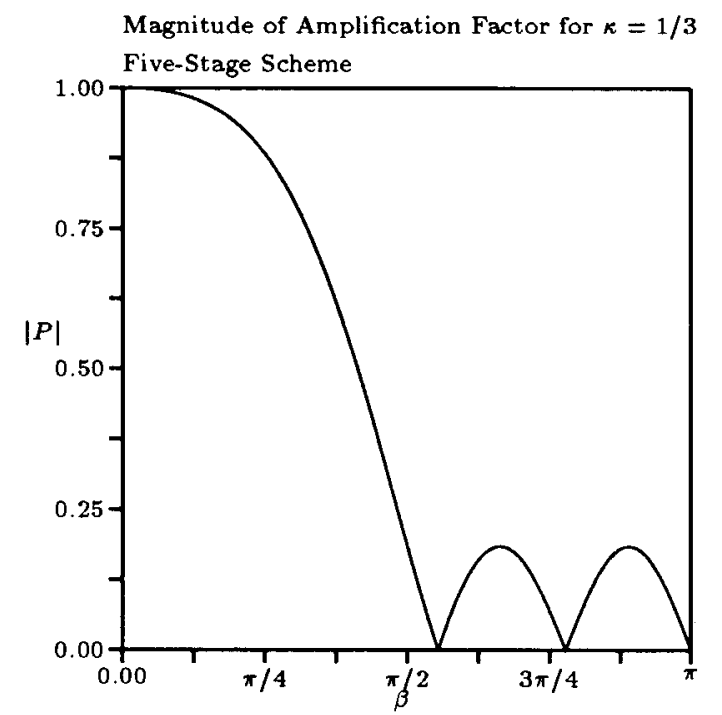

Figure 21: Amplification factor; third-order, five-stage scheme 


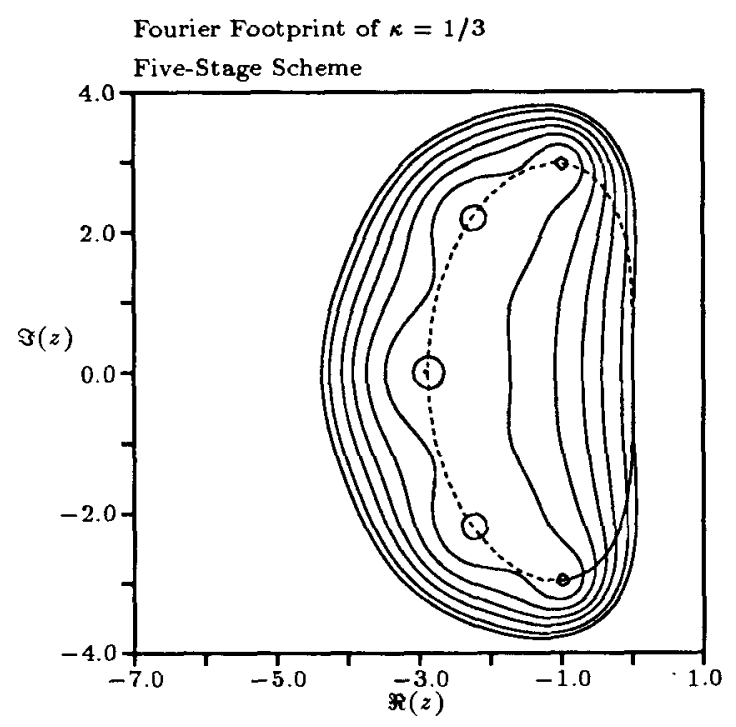

Figure 22: Locus and contours; third-order, five-stage scheme

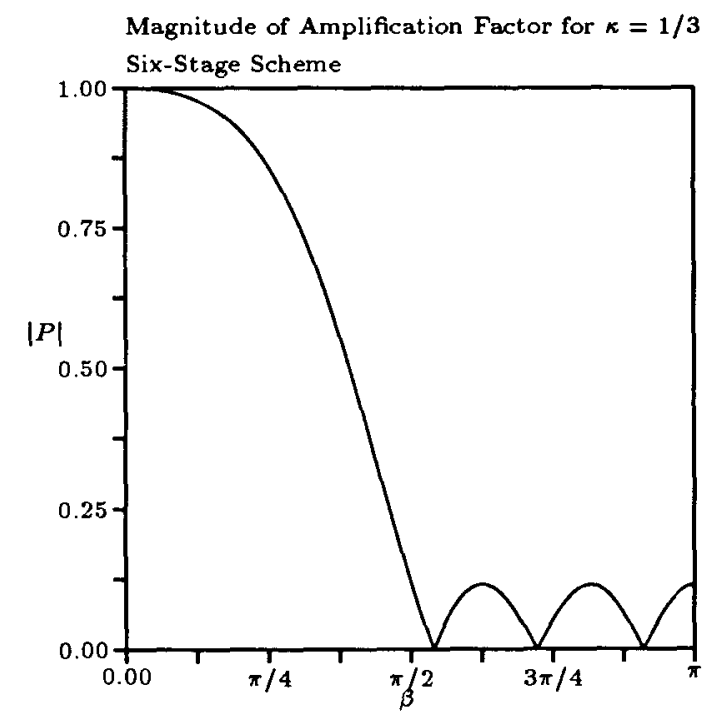

Figure 23: Amplification factor; third-order, six-stage scheme

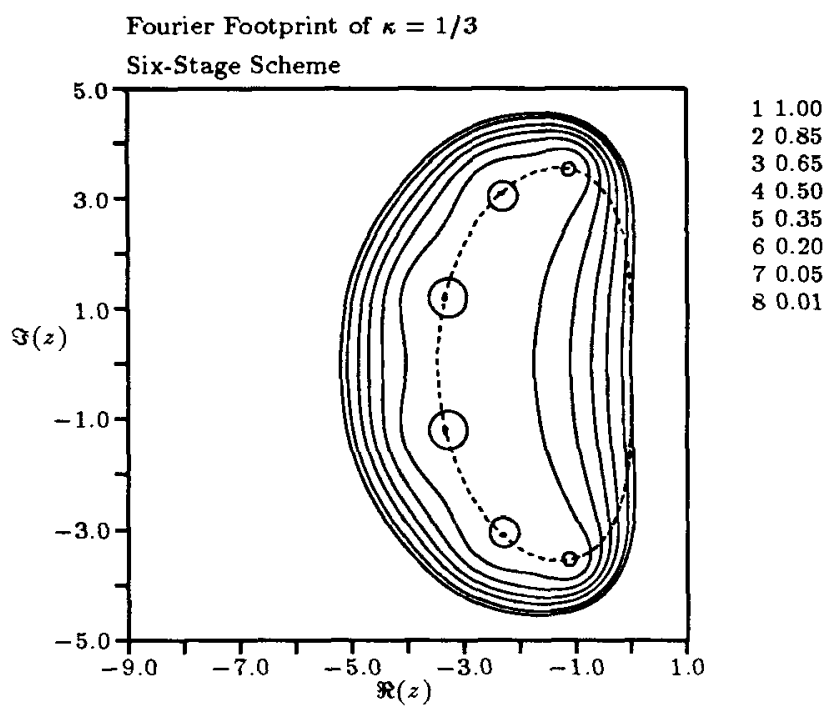

Figure 24: Locus and contours; third-order, six-stage scheme

Number of Stages

\begin{tabular}{|c||c|c|c|c|c|c||}
\hline & 1 & 2 & 3 & 4 & 5 & 6 \\
\hline$\alpha_{1}$ & 1 & 0.3333 & 0.1481 & 0.0833 & 0.0533 & 0.0370 \\
\hline$\alpha_{2}$ & & 1 & 0.4000 & 0.2069 & 0.1263 & 0.0851 \\
\hline$\alpha_{3}$ & & & 1 & 0.4265 & 0.2375 & 0.1521 \\
\hline$\alpha_{4}$ & & & & 1 & 0.4414 & 0.2562 \\
\hline$\alpha_{5}$ & & & & & 1 & 0.4512 \\
\hline$\alpha_{6}$ & & & & & & 1 \\
\hline
\end{tabular}

Table 1: Multi-stage Coefficients for Optimal FirstOrder Scheme

The amplification factor of the above scheme can be written as

$$
\begin{array}{r}
P_{m}(z)=1+z\left(1+\alpha_{m-1} z\left(1+\alpha_{m-2} z(\ldots\right.\right. \\
\left.\left.\left.\ldots\left(1+\alpha_{2} z\left(1+\alpha_{1} z\right)\right) \ldots\right)\right)\right),
\end{array}
$$

where $z$ now corresponds to the CFL number for the full time-interval. The coefficients $\alpha_{k}$ thus are found by multiplying out the string of polynomials of the form 29 or 40 , and re-scaling $z$ such that the linear term gets a coefficient 1.

For example, the three-stage first-order method, as seen from Equations 40 and 48, has amplification factor

$$
P_{3}(z)=\left(1+\frac{z}{2}\right)\left(1+z+\frac{2}{5} z^{2}\right),
$$

with $z$ corresponding to a CFL number of 1 ; it can be 
rewritten as

$$
\begin{aligned}
P_{3}(z) & =1+\frac{3}{2} z+\frac{9}{10} z^{2}+\frac{1}{5} z^{3} \\
& =1+\left(\frac{3}{2} z\right)+\frac{2}{5}\left(\frac{3}{2} z\right)^{2}+\frac{8}{135}\left(\frac{3}{2} z\right)^{3}
\end{aligned}
$$

Number of Stages

\begin{tabular}{|c||c|c|c|c|c||}
\hline & 2 & 3 & 4 & 5 & 6 \\
\hline$\alpha_{1}$ & 0.4242 & 0.1918 & 0.1084 & 0.0695 & 0.0482 \\
\hline$\alpha_{2}$ & 1 & 0.4929 & 0.2602 & 0.1602 & 0.1085 \\
\hline$\alpha_{3}$ & & 1 & 0.5052 & 0.2898 & 0.1885 \\
\hline$\alpha_{4}$ & & & 1 & 0.5060 & 0.3050 \\
\hline$\alpha_{5}$ & & & & 1 & 0.5063 \\
\hline$\alpha_{6}$ & & & & & 1 \\
\hline
\end{tabular}

Table 2: Multi-stage Coefficients for Optimal SecondOrder $(\kappa=-1)$ Scheme or

$$
\begin{aligned}
P_{3}(\bar{z}) & =1+\bar{z}+\frac{2}{5} \bar{z}^{2}+\frac{8}{135} \bar{z}^{3} \\
& =1+\bar{z}\left(1+\frac{2}{5} \bar{z}\left(1+\frac{4}{27} \bar{z}\right)\right),
\end{aligned}
$$

with $\bar{z}=(3 / 2) z$. The scheme is completely defined by specifying

$$
\begin{aligned}
\alpha_{1} & =\frac{4}{27} \\
\alpha_{2} & =\frac{2}{5} \\
\alpha_{3} & =1 \\
\nu & =\frac{3}{2}
\end{aligned}
$$

As mentioned before, the CFL number achieved in an $m$-stage scheme optimized for a particular spatialdifferencing operator is considerably lower than the maximum CFL number that can be realized using any $m$-stage scheme. For the first-order upwind-differencing operator, for instance, the maximum CFL number attainable in $m$ steps equals $m$; in the scheme with optimized high-frequency damping the CFL number amounts to $m / 2$. The minimax values of $|P|$ in the high-frequency range are shown in Table 4; the CFL numbers to achieve these are shown in Table 5.

\section{Application to a Nonlinear Scalar Equation}

A nonlinear convection equation with a source term,

$$
\frac{\partial u}{\partial t}+\frac{\partial}{\partial x}\left(\frac{u^{2}}{2}\right)=\frac{1}{\pi} \sin (\pi x), \quad x \in[0,1],
$$

was chosen for a scalar test of the optimally smoothing multi-stage schemes. The spatial operator was approximated by third-order upwind-biased differencing, corresponding to Equation 64 with $\kappa=1 / 3$. Steady solutions were sought on a grid of 512 cells. Two different kinds of marching-schemes were tried:

1. Runge-Kutta multi-stage schemes, with use of the maximum stable CFL number;

2. Optimally smoothing multi-stage schemes. 


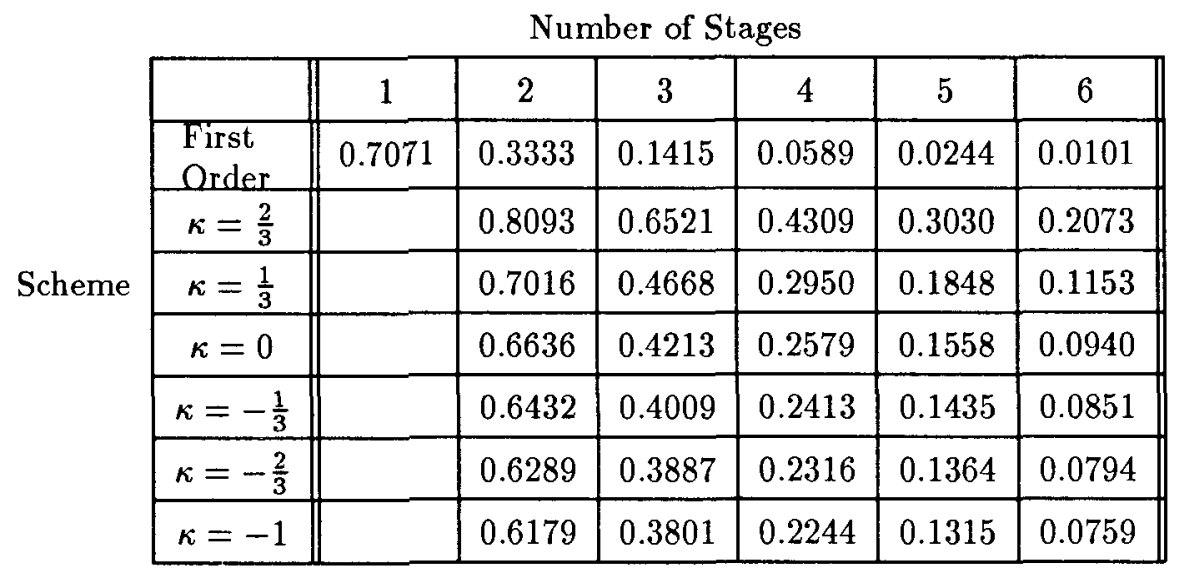

Table 4: $|P|_{\max }$ for $\beta \in[\pi / 2, \pi]$ for Optimal Schemes

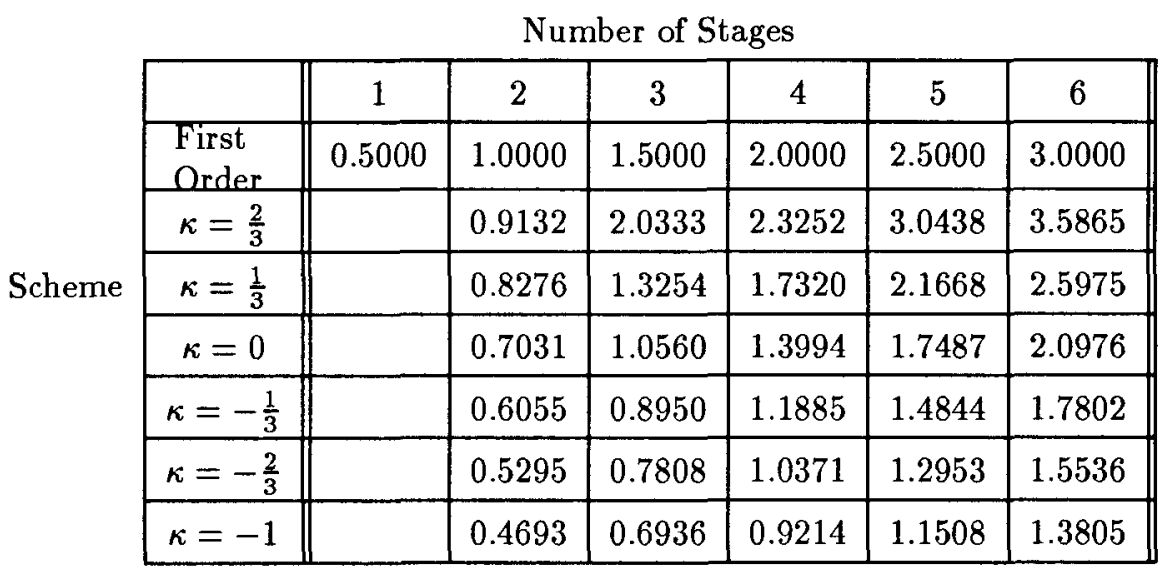

Table 5: Optimal CFL number for Optimal Schemes 
Number of Stages

\begin{tabular}{|c||c|c|c|c|c||}
\hline & 2 & 3 & 4 & 5 & 6 \\
\hline$\alpha_{1}$ & $\frac{1}{2}$ & $\frac{1}{3}$ & $\frac{1}{4}$ & $\frac{1}{5}$ & $\frac{1}{6}$ \\
\hline$\alpha_{2}$ & 1 & $\frac{1}{2}$ & $\frac{1}{3}$ & $\frac{1}{4}$ & $\frac{1}{5}$ \\
\hline$\alpha_{3}$ & & 1 & $\frac{1}{2}$ & $\frac{1}{3}$ & $\frac{1}{4}$ \\
\hline$\alpha_{4}$ & & & 1 & $\frac{1}{2}$ & $\frac{1}{3}$ \\
\hline$\alpha_{5}$ & & & & 1 & $\frac{1}{2}$ \\
\hline$\alpha_{6}$ & & & & & 1 \\
\hline
\end{tabular}

Table 6: Multi-stage Coefficients for Runge-Kutta Schemes

When using the optimally smoothing multi-stage methods, it is crucial to make the CFL number constant over the entire grid, namely, equal to the unique value derived for maximum damping. This amounts to "local time-stepping" at the prescribed CFL number. For the Runge-Kutta schemes, local time-stepping was used at the highest stable CFL numbers. In cells where the convection speed passes through zero, the time-step must be limited (see, e.g. [5]). For both kinds of schemes, a saw-tooth cycle of multi-grid acceleration was used. All solutions were converged to a factor of $10^{-10}$ reduction in the residual.

The coefficients for the first six Runge-Kutta schemes are shown in Table 6; the results of these schemes with regard to convergence speed are summarized in Table 7. The computational work needed for convergence, expressed in terms of finest-grid residual calculations, is shown for various numbers of stages and grid levels. It is clear that the maximum-time-step strategy does not combine with the multi-grid strategy; the reason is that the Runge-Kutta schemes, like most schemes, are not good smoothers at the maximum stable CFL number.

The results of the optimally smoothing schemes are shown in Table 8 . These schemes clearly are a better match for multi-grid acceleration than the RungeKutta schemes. Convergence is reached more quickly in all cases investigated, even on a single grid, despite lower CFL numbers. It is interesting to note that the most efficient of the optimally smoothing schemes, for a sufficient number of grid levels, is the simple two-stage scheme, at least for this simple scalar problem. The gain in smoothing and CFL number achieved with a larger number of stages does not overcome the added computational work.

\begin{tabular}{c|c||c|c|c|c|c||}
\multicolumn{1}{c|}{} \\
\multicolumn{1}{c|}{} & 2 & 3 & 4 & 5 & 6 \\
\hline \multirow{5}{*}{ Grid } & 1 & 1352 & 933 & 816 & 835 & 942 \\
\cline { 2 - 7 } & 2 & 921 & 581 & 528 & 645 & 585 \\
\cline { 2 - 7 } Levels & 3 & 739 & 373 & 350 & 805 & 641 \\
\cline { 2 - 7 } & 4 & 1452 & 315 & 570 & 919 & 642 \\
\cline { 2 - 8 } & 5 & 1585 & 326 & 721 & 969 & 744 \\
\hline & 6 & 2154 & 378 & 749 & 1034 & 839 \\
\hline
\end{tabular}

Table 7: Work required for convergence in scalar case - Runge-Kutta schemes with maximum stable CFL number. Work is expressed as number of finest-grid residual calculations.

\begin{tabular}{c|c||c|c|c|c|c||}
\multicolumn{1}{c|}{} & \multicolumn{6}{c|}{ Number of Stages } \\
\hline & 2 & 3 & 4 & 5 & 6 \\
\hline 1 & 716 & 627 & 660 & 655 & 660 \\
\hline \multirow{4}{*}{ Grid } & 2 & 384 & 365 & 354 & 360 & 369 \\
\cline { 2 - 7 } & 3 & 235 & 221 & 224 & 237 & 252 \\
\cline { 2 - 7 } & 4 & 169 & 180 & 165 & 188 & 203 \\
\cline { 2 - 7 } & 5 & 144 & 198 & 163 & 175 & 186 \\
\hline 6 & 146 & 201 & 166 & 178 & 178 \\
\hline
\end{tabular}

Table 8: Work required for convergence in scalar case - Optimally smoothing schemes. 


\section{Application to a System of Equations}

The next series of numerical experiments was based on the quasi-one-dimensional Euler equations for flow in a converging-diverging channel. Only the optimally smoothing multi-stage schemes were tested. The equations were solved in the form

$$
\frac{\partial}{\partial t}\left(\begin{array}{c}
\rho A \\
\rho u A \\
\rho E A
\end{array}\right)+\frac{\partial}{\partial x}\left(\begin{array}{c}
\rho u A \\
\left(\rho u^{2}+p\right) A \\
\rho u\left(E+\frac{p}{\rho}\right) A
\end{array}\right)=\left(\begin{array}{c}
0 \\
p \frac{d A}{d x} \\
0
\end{array}\right)
$$

where $A$ is the channel area, given by

$$
A(x)=1+\frac{1}{2}(1-\cos (\pi x)), \quad-1 \leq x \leq 1 .
$$

Two test cases were run, both with an inflow Mach number $M_{\infty}=0.3059$ :

1. Shockless transonic flow;

2. Transonic flow with a shock in the diverging portion of the channel.

The Mach number distribution for the second case is shown in Figure 25. In all cases, a sawtooth cycle was used for the multi-grid acceleration, with third-order upwind-biased spatial differencing $(\kappa=1 / 3)$ on the finest grid, and first-order upwind differencing on all coarser grids. In all cases, the parameters associated with the sawtooth cycle (number of solver applications on finest and on coarser grids) were varied in order to obtain convergence in the least amount of work. Each case was run with local time-stepping and characteristic time-stepping for comparison.

The choice of upwind-differencing is not coincidental; in fact it is mandatory for the analysis to extend to the system case. If a conventional approximation is used, based on central-differencing and an artificial viscosity with a scalar coefficient, the loci in the complex plane have a different shape for each wave mode. If upwinddifferencing is used, the loci differ merely by a scale factor. Characteristic time-stepping can then be used to remove these scale factors.

The work required for convergence of the shockless cases, on a fine grid of 256 cells, is indicated in Tables 9 (local time-stepping) and 10 (characteristic timestepping). For local time-stepping, one solver on the finest grid and one solver on the coarser grids was most efficient for all cases. For characteristic time-stepping, with six grid levels, it was advantageous to use two or three solver applications on the finest grid. Even then, the performance of the multi-grid relaxation leveled off for more than four grid levels. The reason for this is not yet understood. It is seen that characteristic time-stepping leads to a substantial improvement
Mach Number Distribution in Channel $\kappa=1 / 3 ; 256$ cells

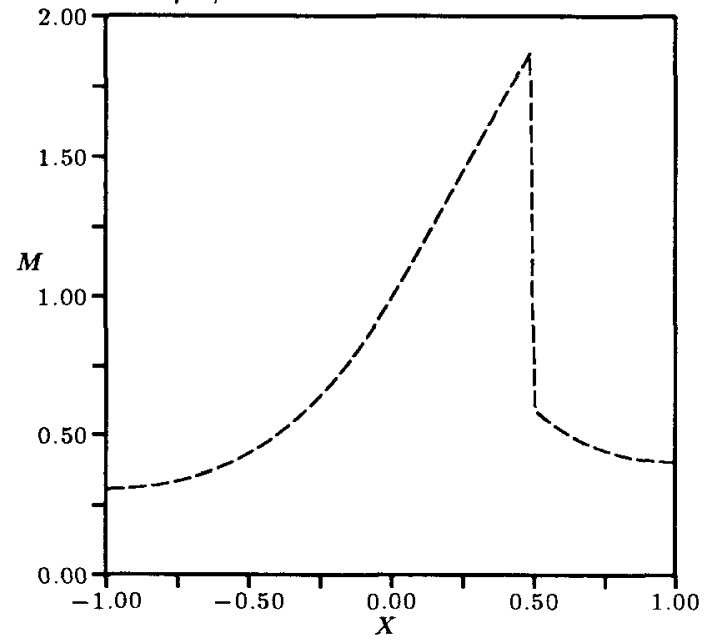

Figure 25: Mach number distribution - Transonic flow with shock

\begin{tabular}{c|c||c|c|c|c|c||}
\multicolumn{1}{c|}{} & \multicolumn{1}{c|}{ Number of Stages } \\
\hline \multicolumn{1}{c}{} & 2 & 3 & 4 & 5 & 6 \\
\hline \multirow{4}{*}{ Grid } & 6455 & 5396 & 5161 & 4952 & 4826 \\
\cline { 2 - 7 } & 2 & 1135 & 1277 & 1441 & 1580 & 1719 \\
\cline { 2 - 7 } & 3 & 822 & 844 & 890 & 938 & 985 \\
\cline { 2 - 7 } & 4 & 795 & 783 & 804 & 832 & 875 \\
\cline { 2 - 7 } & 5 & 871 & 802 & 800 & 797 & 882 \\
\hline 6 & 632 & 589 & 625 & 648 & 718 \\
\hline
\end{tabular}

Table 9: Work required for convergence - Shockless transonic flow - Local time-stepping

in convergence speed over local time-stepping. This is to be expected from the analysis, and can be traced to two causes:

1. Characteristic time-stepping removes stiffness due to the variation among characteristic speeds, thus improving the performance of the scheme on a single grid;

2. By characteristic time-stepping, the optimal CFL number may be used for all waves simultaneously.

The work required for convergence of the cases with a shock is shown in Tables 11 (local time-stepping) and 12 (characteristic time-stepping). For local timestepping, the strategy of one solver application on each grid was the most efficient, except for a few cases with two or three grid levels. These cases required up to 


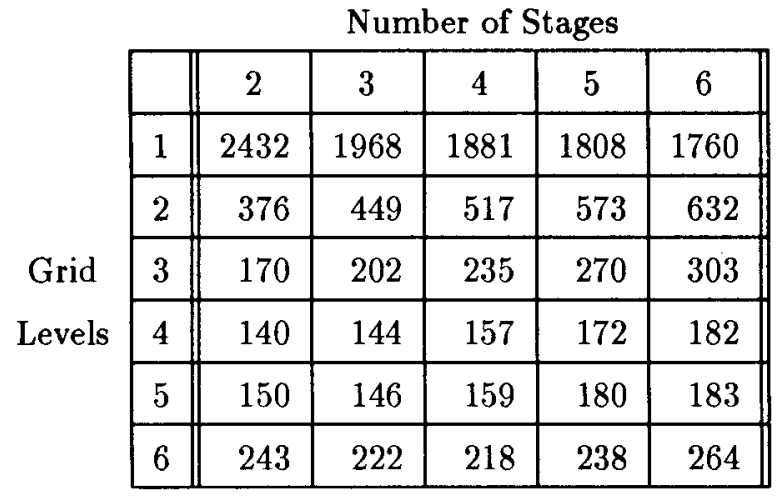

Table 10: Work required for convergence - Shockless transonic flow - Characteristic time-stepping

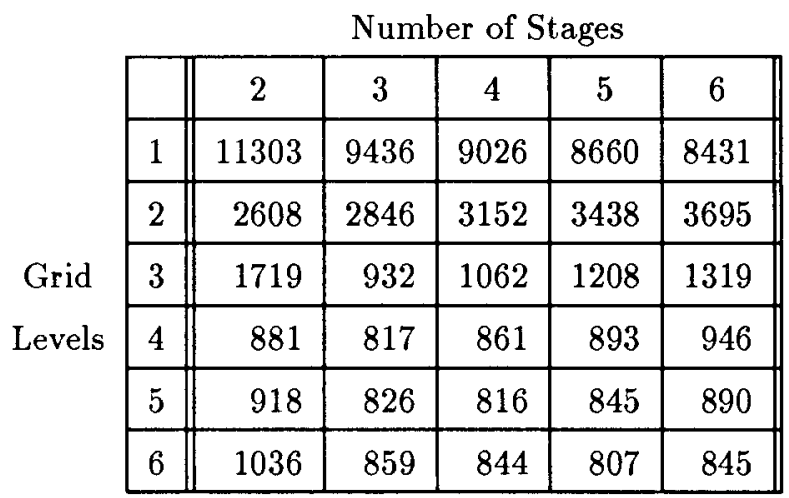

Table 11: Work required for convergence - Transonic flow with shock - Local time-stepping

three solver applications on the finest and/or coarser grids. For characteristic time-stepping, the number of solver applications varied from one to eight on the finest grid, and from one to three on the coarser grids. Even with these variations, four of the cases did not achieve a residual drop of $10^{-10}$, but got hung up between $10^{-5}$ and $10^{-9}$. Nevertheless, in all converged cases, characteristic time-stepping helped speed up convergence.

Figures 26-28 shed some light on the convergence properties of the schemes tested for the shock case. The comparison between local and characteristic timestepping can be seen in Figure 26, which shows the two residual histories for the two-stage scheme with five grid levels. The effect of the number of stages on convergence can be seen in Figure 27, which shows the residual histories for characteristic time-stepping and five grid levels. The effect of the number of grid levels can be seen in Figure 28, which shows the residual histories for the two-stage scheme with characteristic time-stepping.

That the multi-grid convergence is basically independent of the number of cells in the finest grid may be seen

\begin{tabular}{|c|c|c|c|c|c|c|}
\hline & \multicolumn{5}{|c|}{ Number of Stages } \\
\hline & & 2 & 3 & 4 & 5 & 6 \\
\hline \multirow{6}{*}{$\begin{array}{c}\text { Grid } \\
\text { Levels }\end{array}$} & 1 & 6130 & 5200 & 5107 & - & 4584 \\
\hline & 2 & 1589 & 1608 & - & - & - \\
\hline & 3 & 916 & 863 & 987 & 850 & 851 \\
\hline & 4 & 639 & 536 & 526 & 587 & 645 \\
\hline & 5 & 374 & 365 & 390 & 332 & 380 \\
\hline & 6 & 627 & 429 & 464 & 482 & 579 \\
\hline
\end{tabular}

Table 12: Work required for convergence - Transonic flow with shock - Characteristic time-stepping. Dashes denote cases that did not converge to $10^{-10}$.

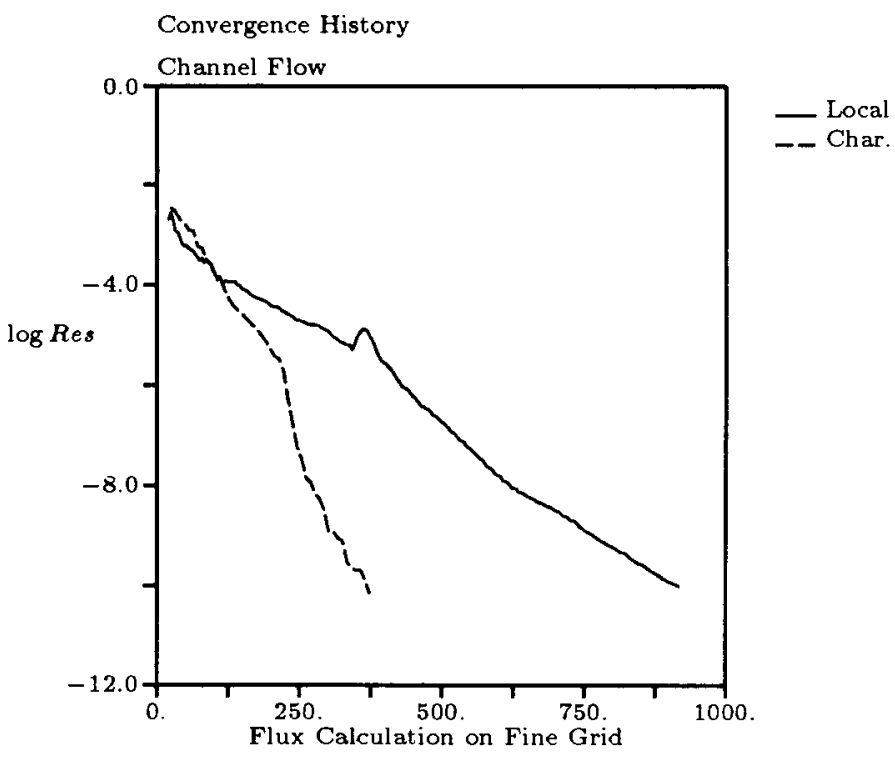

Figure 26: Comparison of local and characteristic timestepping -- Transonic flow with shock - Two stages, five grid levels 


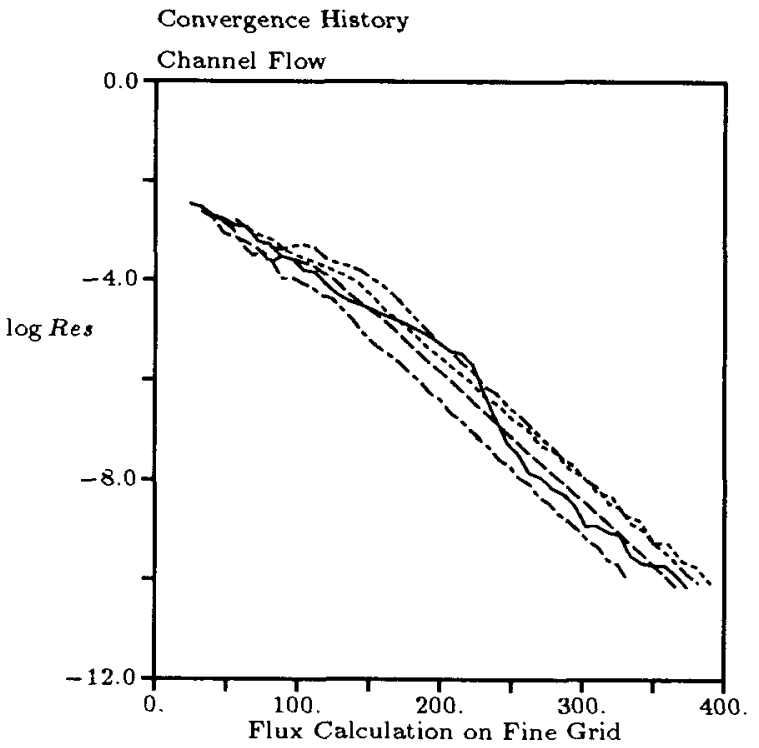

Figure 27: Convergence with different numbers of stages - Transonic flow with shock - Characteristic time-stepping, five grid levels

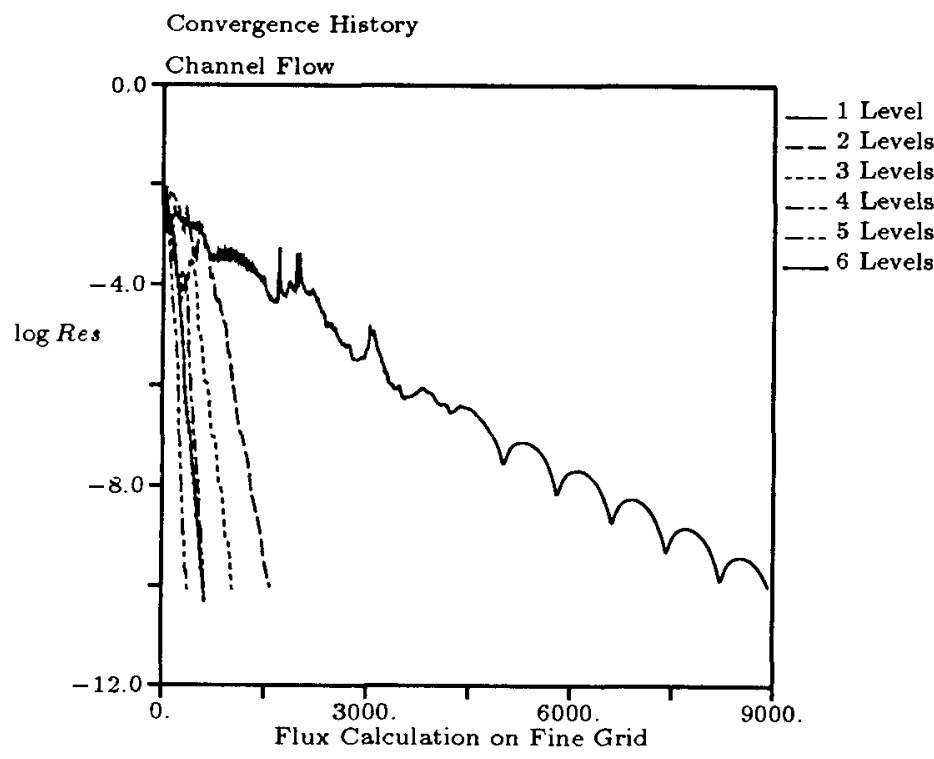

Figure 28: Convergence with different numbers of grid levels - Transonic flow with shock - Characteristic time-stepping, two stages
Convergence History

Channel Flow

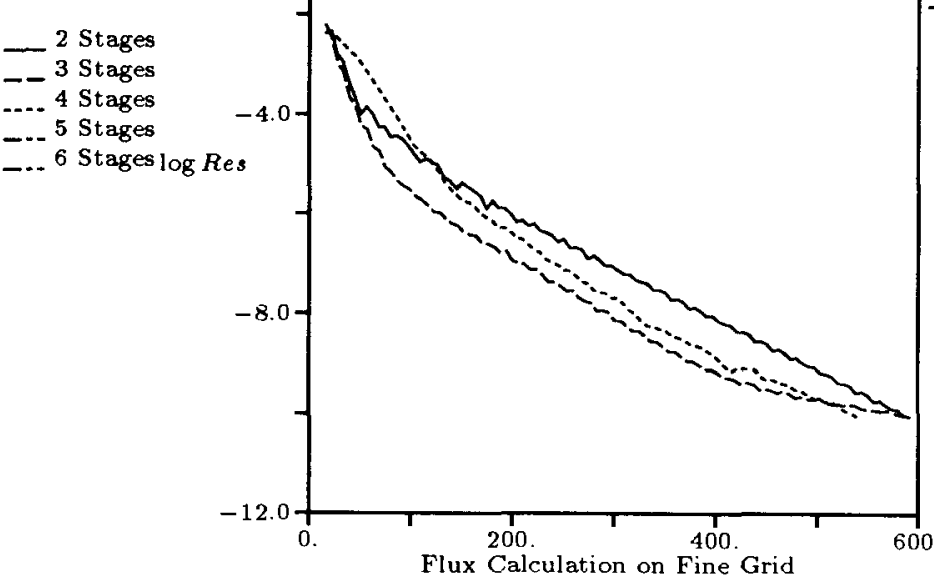

Figure 29: Convergence with different base grids Transonic flow without shock - Local time-stepping, best results per base grid

in Figures 29-32, which show the residual histories for a variety of cases. For each base grid, the numbers of stages and grid levels that gave the best performance were chosen. The convergence histories with characteristic time-stepping (Figures 30 and 32) show the proper behavior, while for local time-stepping, shown in Figures 29 and 31, there is some dependence on the number of cells in the base grid.

\section{Application to Multi-Dimen- sional Equations}

A successful extension of the scalar, one-dimensional analysis to the Euler equations in more than one spacedimension stands or falls with the availability of a robust wave-decomposition model. Work on such decompositions is in progress, but has not yet led to reliable schemes. Without a wave-decomposition model, there is no basis for characteristic time-stepping in more than one space-dimension; the results presented here are for local time-stepping. The test case was a NACA 0012 airfoil at zero incidence in a $M_{\infty}=1.2$ freestream. This case has a bow shock and a fishtail shock. First-order upwind-differencing was used. Table 13 summarizes the number of finest-grid residual calculations necessary for convergence (to $10^{-10}$ ) on a $64 \times 32$ grid. In the sixstage, four grid-level scheme, three iterations on the finest grid followed by one on each of the coarser grids were used; for all other schemes, one iteration on each grid was used. Despite the use of local time-stepping as a substitute for characteristic time-stepping, the multi- 


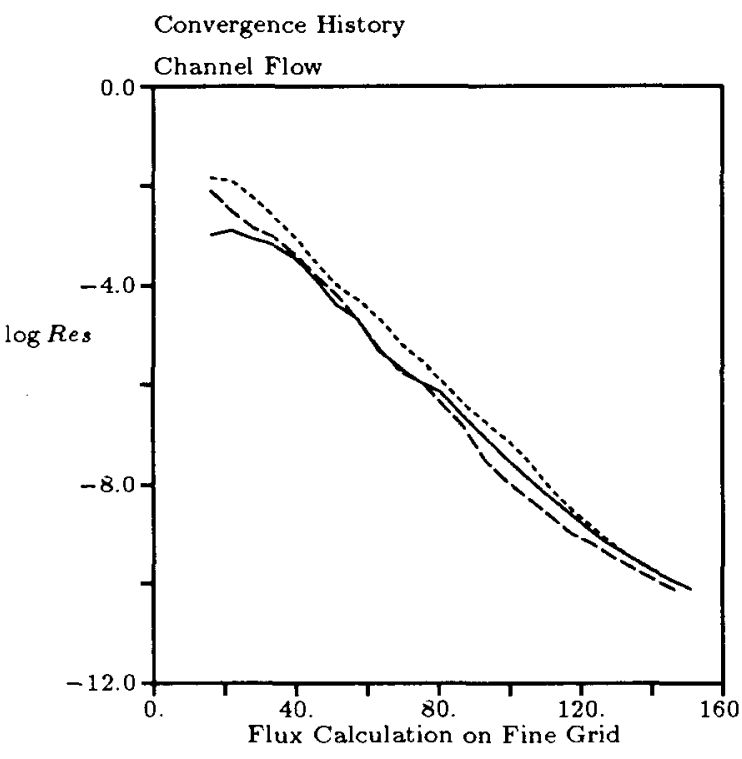

Figure 30: Convergence with different base grids Transonic flow without shock - Characteristic timestepping, best results per base grid

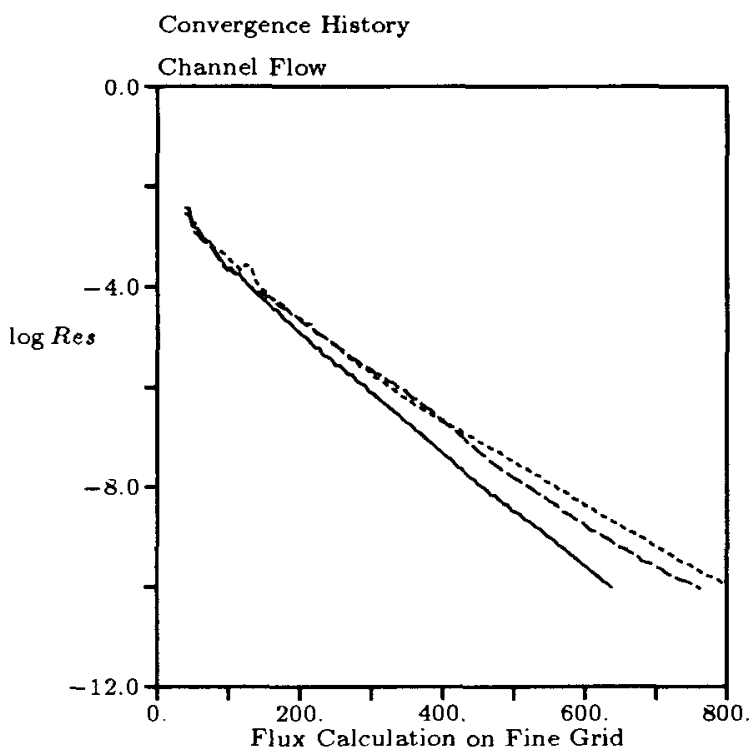

64 Cells --128 Cells .... 256 Cells (256 Cells

Table 13: Work required for convergence - Twodimensional case - Local time-stepping

stage schemes combined well with the multigrid acceleration.

\section{Conclusions and Future Re- search Directions}

In this paper, a method has been developed for designing optimally smoothing multi-stage time-marching schemes, given any spatial-differencing operator. Such schemes are particularly useful in conjunction with multi-grid acceleration. The advantage of using these optimally smoothing schemes has been demonstrated by comparison with Runge-Kutta schemes in solving a nonlinear scalar equation. The analysis has been extended to the Euler equations in one space-dimension by use of characteristic time-stepping. Convergence rates independent of the number of cells in the finest 
grid have been achieved with these optimal schemes, for transonic flow with and without a shock. Besides characteristic time-stepping, local time-stepping has been tested with these schemes. While the analysis is only truly applicable with characteristic time-stepping, good convergence has still been obtained with local timestepping. The extension to two-dimensional flows is hampered by the lack of a robust two-dimensional wave model that may serve as the basis of characteristic timestepping. Again, local time-stepping still led to good convergence.

Future research must concentrate on the important issue of finding a robust two-dimensional wave model that will lead to a robust preconditioning technique. Only with such a technique may full advantage be taken of the optimally smoothing multi-stage schemes.

\section{Acknowledgments}

This work was funded in part by Boeing Commercial Airplane Company, monitored by Dr. Laurence Wigton, and by NASA Langley Research Center under Grant NAG-1-869, monitored by Dr. James Thomas. The authors are indebted to Jos van Kan (Delft University of Technology) for pointing out that the analysis of any multi-stage scheme can be derived from the properties of the two-stage and one-stage schemes.

\section{References}

[1] R. M. Beam and R. F. Warming, "An implicit finite-difference algorithm for hyperbolic systems in conservation law form," Journal of Computational Physics, vol. 22, 1976.

[2] K. G. Powell and E. M. Murman, "An embedded mesh procedure for leading-edge vortex flows," in Proceedings of the Transonic Symposium, 1988.

[3] A. Jameson, W. Schmidt, and E. Turkel, "Numerical solutions of the Euler equations by a finitevolume method using Runge-Kutta time-stepping schemes," AIAA Paper 81-1259, 1981.

[4] E. Turkel, "Preconditioned methods for solving the incompressible and low speed compressible equations." ICASE Report 86-14, 1986.

[5] B. van Leer, W. T. Lee, and K. G. Powell, "Sonicpoint capturing," in AIAA 9th Computational Fluid Dynamics Conference, 1989.

[6] P. L. Roe, "Discrete models for the numerical analysis of time-dependent multidimensional gasdynasmics," Journal of Computational Physics, vol. 63,1986 .
[7] C. Hirsch, C. Lacor, and H. Deconinck, "Convection algorithm based on a diagonalization procedure for the multidimensional Euler equations," in AIAA 8th Computational Fluid Dynamics Conference, 1987.

[8] K. G. Powell and B. van Leer, "A genuinely multidimensional upwind cell-vertex scheme for the Euler Equations," AIAA Paper 89-0095, 1989.

[9] A. Brandt, "Multilevel adaptive computations in fluid dynamics," AIAA Journal, vol. 18, 1980.

[10] A. Jameson, "Numerical solution of the Euler equations for compressible inviscid fluids," in Numerical Methods for the Euler Equations of Fluid Dynamics (F. Angrand, A. Dervieux, J. A. Désidéri, and R. Glowinski, eds.), SIAM, 1985.

[11] B. van Leer, "Upwind-difference methods for aerodynamic problems governed by the Euler equations," in Large-Scale Computations in Fluid Mechanics, Lectures in Applied Mathematics, vol. 22, 1985 . 